\title{
Impact of Integrated Nutrient, Crop Residue and Tillage Management on Soil Aggregates and Organic Matter Fractions in Semiarid Subtropical Soil under Soybean-Wheat Rotation
}

\author{
Milkha S. Aulakh ${ }^{*}$, Ashok K. Garg ${ }^{2}$, Shrvan Kumar ${ }^{3}$ \\ ${ }^{1}$ MSKJ University of Agriculture and Technology, Banda, India; ${ }^{2}$ Krishi Vigyan Kendra, Punjab Agricultural University, Patiala, \\ India; ${ }^{3}$ Department of Soil Science, Punjab Agricultural University, Ludhiana, India. \\ Email: *msaulakh2004@yahoo.co.in
}

Received May 29 $9^{\text {th }}, 2013$; revised June $29^{\text {th }}, 2013$; accepted July $29^{\text {th }}, 2013$

Copyright (C) 2013 Milkha S. Aulakh et al. This is an open access article distributed under the Creative Commons Attribution License, which permits unrestricted use, distribution, and reproduction in any medium, provided the original work is properly cited.

\begin{abstract}
Various physical, chemical and biological soil properties in surface $(0-5 \mathrm{~cm})$ and subsurface $(5-15 \mathrm{~cm})$ soil were determined in a 4-year field experiment conducted at Punjab Agricultural University Ludhiana, India with sixteen treatments consisted of different combinations of fertilizer $\mathrm{N}\left(0,20\right.$ and $\left.25 \mathrm{~kg} \mathrm{~N} \mathrm{ha}^{-1}\right), \mathrm{P}\left(0,60\right.$ and $\left.75 \mathrm{~kg} \mathrm{P}_{2} \mathrm{O}_{5} \mathrm{ha}^{-1}\right)$, FYM $\left(0\right.$ and $\left.10 \mathrm{thha}^{-1}\right)$ and wheat (Triticum aestivum L.) residue (WR) $\left(0\right.$ and $\left.6 \mathrm{t}^{\mathrm{t}} \mathrm{ha}^{-1}\right)$ applied to summer-grown soybean (Glycine max L.) and fertilizer N $\left(0,120\right.$ and $\left.150 \mathrm{~kg} \mathrm{~N} \mathrm{ha}^{-1}\right), \mathrm{P}\left(0,60\right.$ and $\left.75 \mathrm{~kg} \mathrm{P}_{2} \mathrm{O}_{5} \mathrm{ha}^{-1}\right)$, and soybean residue (SR) $(0$ and $3 \mathrm{t} \cdot \mathrm{ha}^{-1}$ ) applied to winter-grown wheat crop continuously in both conventional tillage (CT) and conservation agriculture (CA), arranged in a split-split plot design with tillage system in main blocks, under irrigated subtropical conditions. Application of fertilizer N, P, FYM and crop residue (CR) significantly increased water stable aggregates and had profound effects in increasing the mean weight diameter as well as the formation of macro-aggregates, which were the highest in both surface (85\%) and subsurface (81\%) soil layers with application of $20 \mathrm{~kg} \mathrm{~N}+60 \mathrm{~kg} \mathrm{P}_{2} \mathrm{O}_{5}+10 \mathrm{t} \mathrm{FYM}+$ $6 \mathrm{t} \mathrm{WR} \mathrm{ha}{ }^{-1}$ applied to soybean and $120 \mathrm{~kg} \mathrm{~N}+60 \mathrm{~kg} \mathrm{P}_{2} \mathrm{O}_{5}+3 \mathrm{tSR} \mathrm{ha}^{-1}$ applied to wheat crop in CA, respectively, and were $83 \%$ and $77 \%$ in CT treatments after 2 years. Hence, better aggregation was found with $100 \%$ NP + FYM + CR, where macro-aggregates were greater than $50 \%$ of total soil mass. The same treatment also enhanced total organic $\mathrm{C}$ (TOC) from $3.8 \mathrm{~g} \cdot \mathrm{kg}^{-1}$ in no-NP-FYM-CR control to $5.8 \mathrm{~g} \cdot \mathrm{kg}^{-1}$ in surface layer and from 2.7 to $3.6 \mathrm{~g} \cdot \mathrm{kg}^{-1}$ in subsurface layer after 2 years leading to the $41 \%$ and $39 \%$ higher TOC stocks over CT-Control in $0-15 \mathrm{~cm}$ soil layers of CT and $\mathrm{CA}$, respectively. The changes in TOC stocks after 4 years were $52 \%$ and $59 \%$. Likewise, the labile $\mathrm{C}$ and $\mathrm{N}$ fractions such as water soluble $\mathrm{C}$, particulate and light fraction organic matter, potentially mineralizable $\mathrm{N}$ and microbial biomass were also highest under this integrated inorganic and organic treatment. These results demonstrated that conservation agriculture that integrates application of inorganic fertilizer, organic manure and crop residue, is crucial for improving soil health and sustainability of farming systems in semiarid subtropical soils.
\end{abstract}

Keywords: Soybean; Conservation Agriculture; Water Stable Aggregates; Organic Matter Fractions

\section{Introduction}

Sustainable soil management is desirable to promote profitable agricultural practices that respect the environment, such as conservation agriculture (CA). It attaches great importance to maintenance of soil structure, productivity and biodiversity. It also reduces the negative

"Corresponding author. impacts of tillage, preserves soil resources and can lead to accrual of much of the soil carbon lost during tillage [1]. Intensive land cultivation to meet the food and fiber needs of fast growing population, and removal or burning of crop residues after crop harvest cause losses of organic matter and nutrients from agricultural soils. Burning of crop residues not only results in losses of organic matter and nutrients but also causes atmospheric pollution due 
to the emission of toxic and greenhouse gases like $\mathrm{CO}$, $\mathrm{CO}_{2}$ and $\mathrm{CH}_{4}$ that pose a serious threat to human and environmental health. Soil organic matter (SOM) is important for the supply of $\mathrm{N}, \mathrm{P}$ and $\mathrm{S}$ through mineralization, the retention of some micronutrient elements, enhanced cation exchange capacity, favourable water relations and aggregate stability. Water stable aggregates (WSA) play an important role in nutrient cycling and in supplying substrate for microbial processes that lead to structural stability. However, labile organic matter fractions are readily accessible sources to microorganisms, turnover rapidly (weeks or months), and have direct impact on plant nutrient supply [2]. Labile organic matter fractions generally include water soluble C (WSC), particulate organic matter (POM), light-fraction organic matter (LFOM), soil microbial biomass (SMB), and potentially mineralizable $\mathrm{N}$ (PMN). Microbial biomass $\mathrm{C}$ (MBC) and $\mathrm{N}(\mathrm{MBN})$ generally have higher mineralization rates than SOM. In contrast to temperate climate where decomposition is slow, harsh hot and dry climate in semiarid, arid, subtropical and tropical environments causes fast decomposition. While the soils in these regions are inherently poor in SOM, the use of inadequate and imbalanced chemical fertilizers over a long period adversely affects soil health and productivity [3]. In contrast to CT, CA maintains a continuous soil cover through surface retention of crop residue, with no or reduced tillage, and the use of cover or green manure crops in rotations. However, comparative effects of inorganic fertilizer $\mathrm{N}$ and $\mathrm{P}$, organic manures and crop residue incorporation to crops in CT and CA in semiarid subtropical soils have seldom been investigated. Such information is needed to identify crop nutrient management practices for sustaining or improving soil heath leading to the environmental safety, conservation of resources and the success of sustaining farming systems. Therefore, a 4-year field experiment was conducted to study the changes in soil quality/health parameters like WSA and various organic matter fractions under CA and CT systems.

\section{Materials and Methods}

\subsection{Experimental Site and Climate}

A 4-year field experiment on soybean-wheat rotation was established in 2005 at Punjab Agricultural University Research Farm, Ludhiana $\left(30^{\circ} 54^{\prime} \mathrm{N}, 75^{\circ} 48^{\prime} \mathrm{E}, 247 \mathrm{~m}\right.$ above mean sea level), India on a Fatehpur loamy sand soil (Typic Haplustept). During the 4-year period of field experiment, while the mean monthly minimum air temperature ranged from $4^{\circ} \mathrm{C}$ in January to $28^{\circ} \mathrm{C}$ in July, maximum temperature ranged from $17^{\circ} \mathrm{C}$ in January to $40^{\circ} \mathrm{C}$ in June. The annual rainfall ranged from 563 to 995 mm of which $71 \%$ to $88 \%$ was received during monsoon period (June-September).

\subsection{Treatments and Field Operations}

A total of 16 treatment combinations in triplicate with respect to CT and CA system, crop residue and fertilizer $\mathrm{N}$ and $\mathrm{P}$ (as listed in Table 1) were arranged in a split-split plot design with tillage system in main blocks, CR treatments in sub plots and fertilizer treatments in sub-sub plots of $3.15 \times 8.30 \mathrm{~m}$ size. The recommended rates of fertilizer nutrients for optimum yields of soybean are $20 \mathrm{~kg} \mathrm{~N}$ and $60 \mathrm{~kg} \mathrm{P}_{2} \mathrm{O}_{5} \mathrm{ha}^{-1}$ [4] and wheat are 120 $\mathrm{kg} \mathrm{N}$ and $60 \mathrm{~kg} \mathrm{P}_{2} \mathrm{O}_{5} \mathrm{ha}^{-1}$. Experimental details and results on crop yields, nutrients uptake etc. from this experiment have been reported earlier [5]. In brief, the treatments consisted of control $\left(\mathrm{T}_{1}\right), 100 \%$ of recommended NP without CR $\left(\mathrm{T}_{2}\right), 125 \%$ of recommended NP without $\mathrm{CR}\left(\mathrm{T}_{3}\right), 100 \% \mathrm{NP}+10 \mathrm{t} \mathrm{FYM} \mathrm{ha}^{-1}$ without $\mathrm{CR}$ $\left(\mathrm{T}_{4}\right)$ and each of these treatments with $\mathrm{CR}\left(\mathrm{T}_{5}, \mathrm{~T}_{6}, \mathrm{~T}_{7}\right.$ and $\mathrm{T}_{8}$ ) in CT. The corresponding treatments were given in CA as well ( $T_{9}$ to $T_{16}$, respectively). In CR treatments, $6 \mathrm{t}$ wheat residue $\mathrm{ha}^{-1}$ and $3 \mathrm{t}$ soybean residue $\mathrm{ha}^{-1}$ were incorporated in $\mathrm{CT}$ and spread on the soil surface in CA system. While the soil was tilled in CT treatments, crops were seeded by using manually-operated plough minimizing the soil disturbance in CA. At harvesting of each crop, the residue was either removed or incorporated in respective treatments in CT. In respective treatments of CA, crop residue was either removed or retained on the soil surface. In crop residue treatments, wheat residue was incorporated in $\mathrm{CT}$ and spread on the soil surface in CA system before seeding soybean. Similarly, soybean residue was applied before sowing wheat. The crops were irrigated $(7.5 \mathrm{~cm})$ as and when required by taking into consideration the moisture received through rainfall.

\subsection{Soil Sampling and Analyses}

After the completion of two and four cycles of soybean-wheat rotations, representative soil samples were collected from four random spots within each plot and mixed thoroughly to prepare a composite sample for 0 - 5 and $5-15 \mathrm{~cm}$ layer and air dried under shade. A portion of each sample was passed through $2 \mathrm{~mm}$ sieve and analyzed for TOC, water soluble C (WSC), particulate organic $\mathrm{C}$ and $\mathrm{N}$ (POC and $\mathrm{PON}$ ), light fraction organic $\mathrm{C}$ and $\mathrm{N}$ (LFOC and LFON), potentially mineralizable $\mathrm{N}$ $(\mathrm{PMN}$ ), and microbial biomass $\mathrm{C}$ and $\mathrm{N}$ (MBC and MBN). Rest of the clods was broken by hand in such a manner that the size of the clods remained between $5-8$ $\mathrm{mm}$, later were used for the estimation of water stable aggregates (WSA). The aggregate size distribution was determined by wet sieving method as described by Yoder 
Table 1. Effect of fertilizer, FYM and crop residue treatments on soil aggregate size distribution (\%) and mean weight diameter (MWD) in 0 - $5 \mathrm{~cm}$ soil layer after 2-year of the experiment.

\begin{tabular}{|c|c|c|c|c|c|c|c|c|c|}
\hline \multirow{2}{*}{$\begin{array}{c}\text { Treatment } \\
\text { No. }\end{array}$} & \multicolumn{2}{|c|}{ Treatments } & \multicolumn{7}{|c|}{ Distribution of WSA in different sizes (\%) } \\
\hline & Soybean & Wheat & $0.11-0.25 \mathrm{~mm}$ & $0.25-0.50 \mathrm{~mm}$ & $0.5-1.0 \mathrm{~mm}$ & $1.0-2.0 \mathrm{~mm}$ & $>2.0 \mathrm{~mm}$ & Total WSA (\%) & $\begin{array}{l}\text { MWD } \\
(\mathrm{mm})\end{array}$ \\
\hline \multicolumn{10}{|c|}{ Conventional tillage } \\
\hline $\mathrm{T}_{1}$ & $\mathrm{~N}_{0} \mathrm{P}_{0} \mathrm{WR}_{0}^{\dagger}$ & $\mathrm{N}_{0} \mathrm{P}_{0} \mathrm{SR}_{0}^{\ddagger}$ & 23.2 & 22.7 & 14.9 & 7.1 & 3.1 & 70.9 & 0.41 \\
\hline $\mathrm{T}_{2}$ & $\mathrm{~N}_{20} \mathrm{P}_{60} \mathrm{WR}_{0}$ & $\mathrm{~N}_{120} \mathrm{P}_{60} \mathrm{SR}_{0}$ & 25.2 & 25.4 & 14.3 & 9.6 & 4.5 & 79.0 & 0.44 \\
\hline $\mathrm{T}_{3}^{\S}$ & $\mathrm{N}_{25} \mathrm{P}_{75} \mathrm{WR}_{0}$ & $\mathrm{~N}_{150} \mathrm{P}_{75} \mathrm{SR}_{0}$ & - & - & - & - & - & - & - \\
\hline $\mathrm{T}_{4}$ & \multicolumn{2}{|c|}{$\mathrm{N}_{20} \mathrm{P}_{60} \mathrm{WR}_{0}+\mathrm{FYM}_{10} \mathrm{~N}_{120} \mathrm{P}_{60} \mathrm{SR}_{0}$} & 24.7 & 25.5 & 14.7 & 10.2 & 5.6 & 80.6 & 0.47 \\
\hline $\mathrm{T}_{5}$ & $\mathrm{~N}_{0} \mathrm{P}_{0} \mathrm{WR}_{6}$ & $\mathrm{~N}_{0} \mathrm{P}_{0} \mathrm{SR}_{3}$ & 25.9 & 20.9 & 15.5 & 7.6 & 3.8 & 73.7 & 0.42 \\
\hline $\mathrm{T}_{6}$ & $\mathrm{~N}_{20} \mathrm{P}_{60} \mathrm{WR}_{6}$ & $\mathrm{~N}_{120} \mathrm{P}_{60} \mathrm{SR}_{3}$ & 25.5 & 22.6 & 16.5 & 11.9 & 5.2 & 81.7 & 0.48 \\
\hline $\mathrm{T}_{7}^{\S}$ & $\mathrm{N}_{25} \mathrm{P}_{75} \mathrm{WR}_{6}$ & $\mathrm{~N}_{150} \mathrm{P}_{75} \mathrm{SR}_{3}$ & - & - & - & - & - & - & - \\
\hline \multirow[t]{3}{*}{$\mathrm{T}_{8}$} & \multicolumn{2}{|c|}{$\mathrm{N}_{20} \mathrm{P}_{60} \mathrm{WR}_{6}+\mathrm{FYM}_{10} \mathrm{~N}_{120} \mathrm{P}_{60} \mathrm{SR}_{3}$} & 25.0 & 22.8 & 17.3 & 12.8 & 5.3 & 83.3 & 0.49 \\
\hline & \multicolumn{2}{|l|}{ CT Mean } & 24.9 & 23.3 & 15.5 & 9.9 & 4.6 & 78.2 & 0.45 \\
\hline & \multicolumn{2}{|l|}{$L S D(0.05)$} & ns & ns & 0.78 & 0.87 & 0.69 & 1.7 & 0.01 \\
\hline & \multicolumn{9}{|c|}{ Conservation agriculture } \\
\hline $\mathrm{T}_{9}$ & $\mathrm{~N}_{0} \mathrm{P}_{0} \mathrm{WR}_{0}$ & $\mathrm{~N}_{0} \mathrm{P}_{0} \mathrm{SR}_{0}$ & 22.1 & 23.9 & 14.1 & 10.1 & 4.9 & 75.1 & 0.47 \\
\hline $\mathrm{T}_{10}$ & $\mathrm{~N}_{20} \mathrm{P}_{60} \mathrm{WR}_{0}$ & $\mathrm{~N}_{120} \mathrm{P}_{60} \mathrm{SR}_{0}$ & 24.8 & 25.1 & 15.3 & 10.6 & 5.3 & 81.1 & 0.47 \\
\hline $\mathrm{T}_{11}^{\S}$ & $\mathrm{N}_{25} \mathrm{P}_{75} \mathrm{WR}_{0}$ & $\mathrm{~N}_{150} \mathrm{P}_{75} \mathrm{SR}_{0}$ & - & - & - & - & - & - & - \\
\hline $\mathrm{T}_{12}$ & \multicolumn{2}{|c|}{$\mathrm{N}_{20} \mathrm{P}_{60} \mathrm{WR}_{0}+\mathrm{FYM}_{10} \mathrm{~N}_{120} \mathrm{P}_{60} \mathrm{SR}_{0}$} & 23.6 & 27.5 & 15.3 & 10.5 & 5.0 & 81.9 & 0.46 \\
\hline $\mathrm{T}_{13}$ & $\mathrm{~N}_{0} \mathrm{P}_{0} \mathrm{WR}_{6}$ & $\mathrm{~N}_{0} \mathrm{P}_{0} \mathrm{SR}_{3}$ & 24.0 & 20.9 & 15.2 & 10.6 & 5.1 & 75.8 & 0.48 \\
\hline $\mathrm{T}_{14}$ & $\mathrm{~N}_{20} \mathrm{P}_{60} \mathrm{WR}_{6}$ & $\mathrm{~N}_{120} \mathrm{P}_{60} \mathrm{SR}_{3}$ & 26.7 & 23.4 & 15.6 & 11.2 & 5.5 & 82.6 & 0.47 \\
\hline $\mathrm{T}_{15} \S$ & $\mathrm{N}_{25} \mathrm{P}_{75} \mathrm{WR}_{6}$ & $\mathrm{~N}_{150} \mathrm{P}_{75} \mathrm{SR}_{3}$ & - & - & - & - & - & - & - \\
\hline \multirow[t]{3}{*}{$\mathrm{T}_{16}$} & \multicolumn{2}{|c|}{$\mathrm{N}_{20} \mathrm{P}_{60} \mathrm{WR}_{6}+\mathrm{FYM}_{10} \mathrm{~N}_{120} \mathrm{P}_{60} \mathrm{SR}_{3}$} & 24.6 & 27.7 & 15.6 & 11.4 & 5.4 & 84.7 & 0.47 \\
\hline & \multicolumn{2}{|c|}{ CA Mean } & 24.3 & 24.8 & 15.2 & 10.7 & 5.2 & 80.2 & 0.47 \\
\hline & \multicolumn{2}{|l|}{$L S D(0.05)$} & ns & 3.7 & 0.8 & ns & ns & 2.8 & ns \\
\hline
\end{tabular}

${ }^{\dagger} \mathrm{N}=$ fertilizer $\mathrm{N}\left(\mathrm{kg} \mathrm{N} \mathrm{ha}^{-1}\right) ; \mathrm{P}=$ fertilizer $\mathrm{P}\left(\mathrm{kg} \mathrm{P}_{2} \mathrm{O}_{5} \mathrm{ha}^{-1}\right) ; \mathrm{WR}=$ Wheat crop residue $\left(\mathrm{t} \cdot \mathrm{ha}^{-1}\right) ;{ }^{*} \mathrm{SR}=$ Soybean crop residue $\left(\mathrm{t} \cdot \mathrm{ha}{ }^{-1}\right) ;{ }^{8} \mathrm{WSA}$ and $\mathrm{MWD}$ were not measured in Treatments $\left(\mathrm{T}_{3}, \mathrm{~T}_{7}, \mathrm{~T}_{11}\right.$, and $\left.\mathrm{T}_{15}\right) ; \mathrm{FYM}=$ farmyard manure $\left(\mathrm{t} \cdot \mathrm{ha}^{-1}\right) ; \mathrm{ns}=$ non-significant.

[6] by uniformly spreading soil clods on the top most sieve of a nest of sieves having pore diameter $2,1,0.5$, 0.25 and $0.11 \mathrm{~mm}$. The nest of sieves was oscillated up and down by a pulley arrangement for 30 minutes at a frequency of 30 cycles per minute in salt-free water. WSA of different sizes were collected from the respective sieves separately after oven-drying the sieves at $50^{\circ} \mathrm{C}$.

$\% \mathrm{WSA}$ in each size group $=($ Weight of aggregates in each size group / Total weight of soil) $\times 100$

Total $\mathrm{N}$ content of soil samples was determined by digestion in concentrated $\mathrm{H}_{2} \mathrm{SO}_{4}$ followed by Kjeldahl's steam distillation. WSC was determined by following the method described by McGill et al. [7] and POC and PON were determined by the method of Cambardella and Elliott [8]. LFOC and LMON were determined by the method of Compton and Boone [9], and PMN in soil was determined by following the incubation method [10]. The chloroform fumigation and incubation method was used for the determination of biomass in the soil [11]. For this, the soil samples were pre-incubated for 7 days at $40 \%$ WHC at $25^{\circ} \mathrm{C}$ to avoid the exaggerated values as there is spurt of microbial activity in soil after wetting of dry soil [12]. The moisture content of the soil samples was adjusted to $55 \%$ WHC by adding sterile distilled water. The increases in $\mathrm{CO}_{2}$ evolution and extractable $\mathrm{NH}_{4}^{+}$from fumigated samples were determined to estimate the MBC and MBN, respectively. In the representative soil samples collected from $0-5$ and $5-15 \mathrm{~cm}$ layers after 4 years of experimentation, soil $\mathrm{pH}$ was determined in soil:water suspension (1:2 ratio) using glass electrode.

\subsection{Statistical Analysis}

Statistical analysis of data of WSA and various soil health parameters was carried out by ANOVA in split-split plot design [13]. The effects of different treat- 
ments were evaluated using the least significant difference (LSD) test at the 0.05 level of probability. The data presented in figures are means \pm standard deviation (SD) of three replications.

\section{Results}

\subsection{Soil Physical Properties}

\subsubsection{Water Stable Aggregates}

Results on total WSA after 2 years of the experiment showed that in $0-5 \mathrm{~cm}$ soil layer of CT system, $\mathrm{T}_{2}$ and $\mathrm{T}_{4}$ treatments increased total WSA from $71 \%$ in control $\left(\mathrm{T}_{1}\right)$ to 79 and $81 \%$ without $\mathrm{CR}$, and to $82\left(\mathrm{~T}_{6}\right)$ and $83 \%$ $\left(\mathrm{T}_{8}\right)$ with CR, respectively (Figure 1). The corresponding increase of total WSA under CA system was $75 \%$ in control $\left(\mathrm{T}_{9}\right)$ to $81\left(\mathrm{~T}_{10}\right)$ and $82 \%\left(\mathrm{~T}_{12}\right)$ without $\mathrm{CR}$ and 83 $\left(\mathrm{T}_{14}\right)$ and $85 \%\left(\mathrm{~T}_{16}\right)$ in with CR, respectively. In $5-15$ $\mathrm{cm}$ layer, the increasing trends due to the application of $\mathrm{N}$ and $\mathrm{P}$ fertilizers, FYM and CR were similar to those observed in $0-5 \mathrm{~cm}$ layer, however, the magnitude was relatively lower (Figure 2). The impact of applied fertilizer, FYM and CR in improving total WSA was significant in $0-5 \mathrm{~cm}$ soil layer, and higher than in $5-15 \mathrm{~cm}$ soil layer under both $\mathrm{CA}$ and $\mathrm{CT}$ system. The trends were similar after 4 cycles of the experiment with small increase in WSA.

\subsubsection{Distribution of Aggregates in Different Size}

Distribution of aggregates in different size classes $(0.11$ $0.25,0.25-0.5,0.5-1.0,1-2$ and $>2$ mm diameter) in two soil layers under CT and CA system is presented in Tables 1 and 2. In all the treatments, the proportion of macro-aggregates in the size class of 0.25 to $>2 \mathrm{~mm}$ was higher as compared to micro-aggregate in the size class $0.11-0.25 \mathrm{~mm}$. Among the macro-aggregates, $0.25-$ $0.50 \mathrm{~mm}$ fraction constituted the greatest proportion followed by $0.5-1.0,1.0-2.0$, and $>2 \mathrm{~mm}$ fraction constituted the least proportion in both $0-5 \mathrm{~cm}$ and $5-15 \mathrm{~cm}$

\section{After 2 years}

Without CR

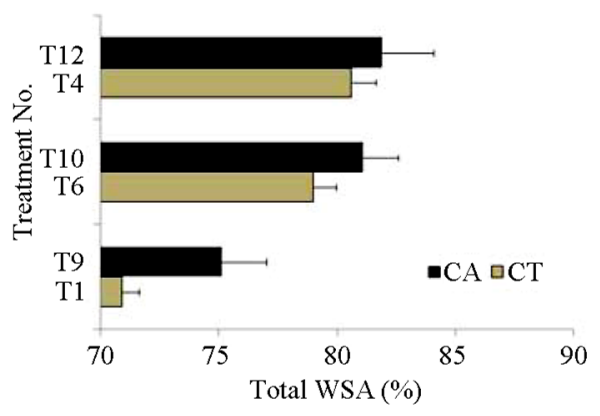

$\underline{\text { With CR }}$

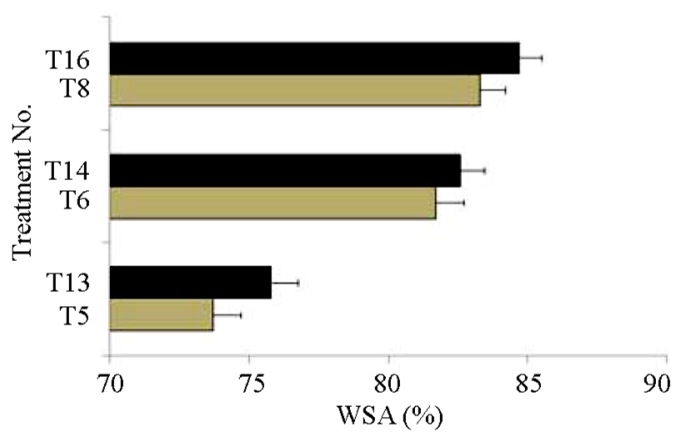

$\underline{\text { After } 4 \text { years }}$

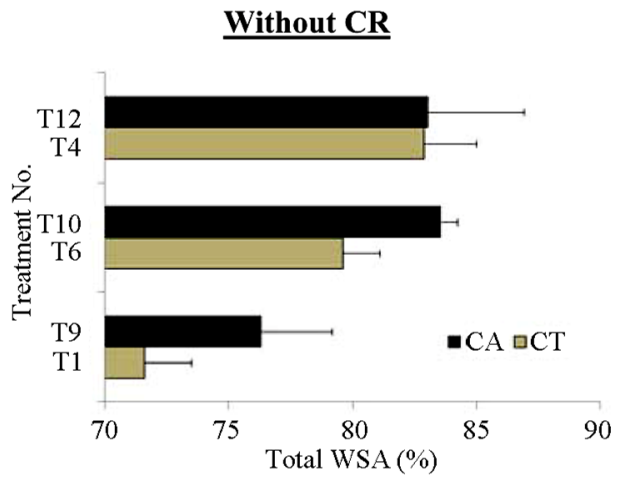

With CR

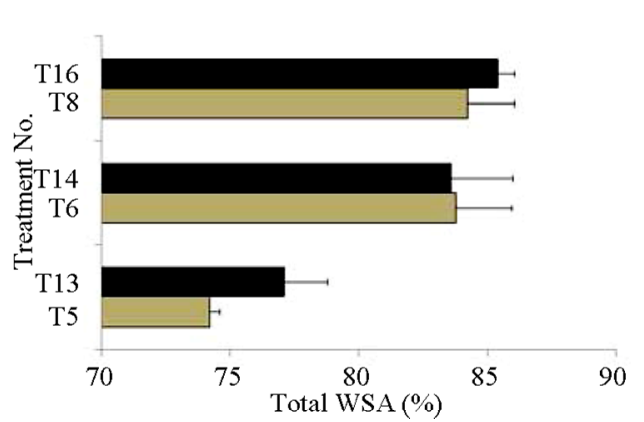

Figure 1. Effect of fertilizer, tillage and crop residue treatments on total water stable aggregate (WSA) in 0 - 5 cm soil layer after 2-year and 4-year of soybean-wheat experiment under CT (Grey color bars) and CA systems (Black color bars). For treatment abbreviations, see Table 1. 


\section{$\underline{\text { After } 2 \text { years }}$}
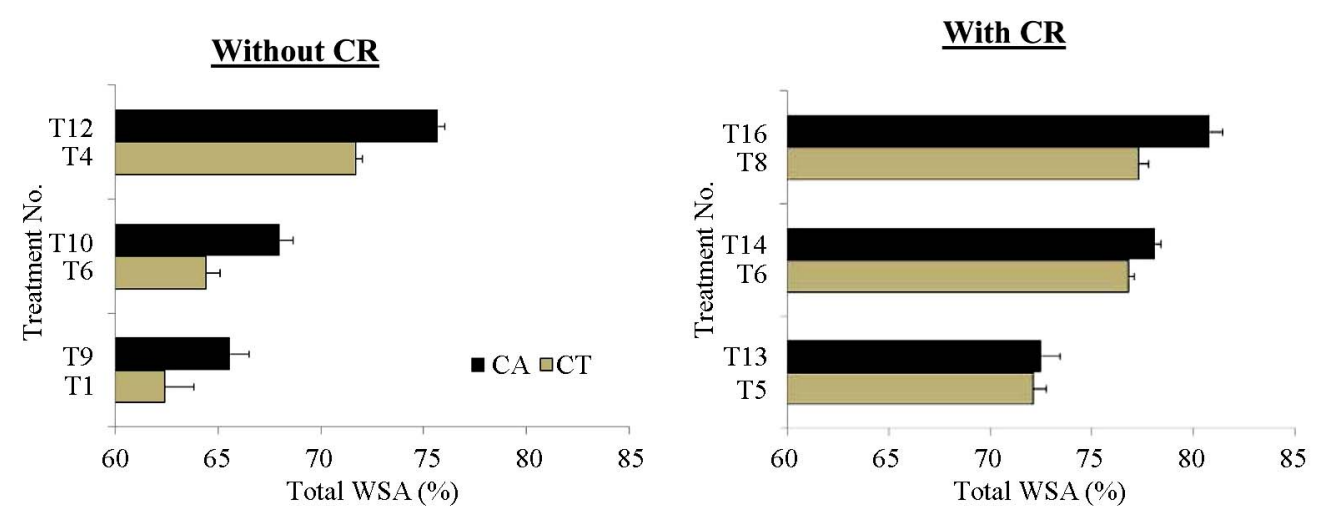

\section{$\underline{\text { After } 4 \text { years }}$}
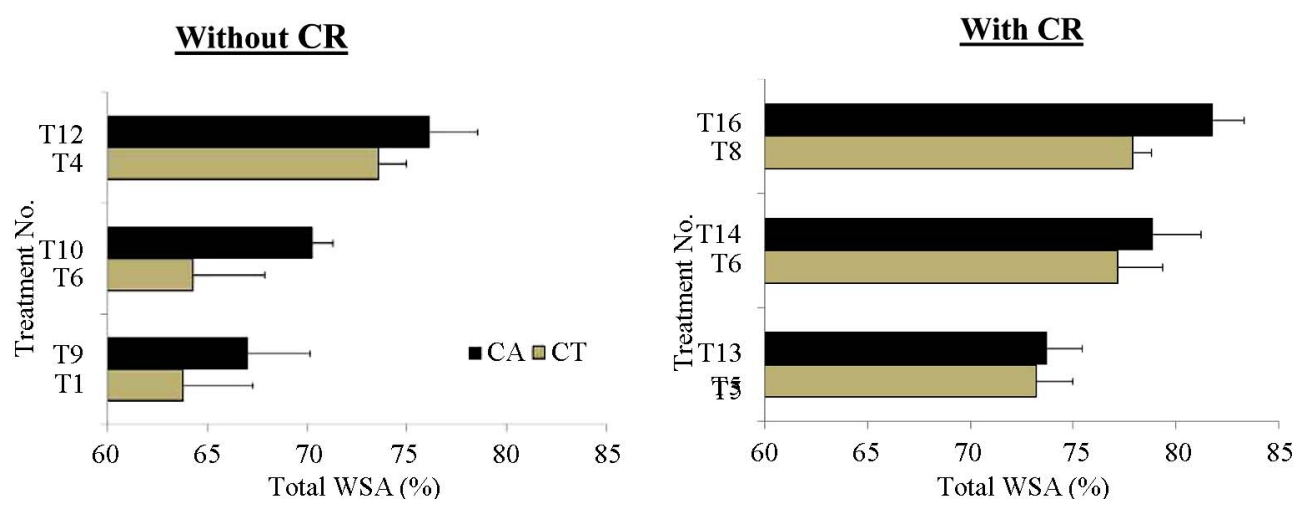

Figure 2. Effect of fertilizer, tillage and crop residue treatments on total water stable aggregate (WSA) in 5 - 15 cm soil layer after 2-year and 4-year of soybean-wheat experiment under CT and CA systems (For treatment abbreviations, see Table 1).

soil layers under both $\mathrm{CT}$ and CA system. Integrated use of organic and inorganic fertilizers significantly increased total WSA which was highest in all the macro-aggregate size fractions in $0-5 \mathrm{~cm}$ (Table 1) and $5-15 \mathrm{~cm}$ soil layer (Table 2). In $0-5 \mathrm{~cm}$ soil layer of CA system without CR, macro-aggregates in the 0.25 $0.5,0.5-1.0,1-2$, and $>2 \mathrm{~mm}$ were $23.9,14.1,10.1$ and $4.9 \%$, respectively in control plots $\left(\mathrm{T}_{9}\right)$, which increased to $27.5,15.3,10.5$ and $5.0 \%$, respectively in $\mathrm{T}_{12}$ plots. Whereas with $\mathrm{CR}$, macro-aggregate in the respective classes were 20.9, 15.2, 10.6 and 5.1\%, in control $\left(\mathrm{T}_{13}\right)$, which increased to 27.7, 15.6, 11.4 and $5.4 \%$ in $\mathrm{T}_{16}$ treatment. In $5-15 \mathrm{~cm}$ soil layer, the increasing trends of macro-aggregates in these classes under CT and CA system due to the application of $\mathrm{N}$ and $\mathrm{P}$ fertilizers, FYM and $\mathrm{CR}$ were quite similar to those in $0-5 \mathrm{~cm}$ layer, however, the magnitude was relatively lower (Table 2).

Micro-aggregate $(0.11-0.25 \mathrm{~mm})$ ranged from $23 \%$ $26 \%$ in the $0-5 \mathrm{~cm}$ (Table 1) and $21-24 \%$ in $5-15 \mathrm{~cm}$ soil layer in CT (Table 2). Whereas under CA system, micro-aggregate ranged from $22 \%-27 \%$ in $0-5 \mathrm{~cm}$ layer (Table 1) and $21 \%-25 \%$ in the $5-15 \mathrm{~cm}$ soil layer (Table 2).

MWD in the $0-5 \mathrm{~cm}$ layer ranged from 0.41 to 0.49 $\mathrm{mm}$ in CT and 0.46 to $0.48 \mathrm{~mm}$ in CA system (Table 1). The corresponding values for $5-15 \mathrm{~cm}$ soil layer varied from 0.41 to 0.51 and 0.43 to $0.48 \mathrm{~mm}$ (Table 2). The MWD was significantly higher in FYM and CR treatments as compared to control in CT system in both the soil layers.

\subsection{Soil Chemical Properties}

\subsubsection{Total Organic $\mathbf{C}$, Total $\mathbf{N}$ and $\mathbf{C} / \mathbf{N}$ Ratio}

Results on TOC content after 2 and 4 years showed that with $\mathrm{N}$ and $\mathrm{P}$ fertilizers, FYM and CR treatments, TOC content significantly differed under CT and CA systems in both $0-5 \mathrm{~cm}$ and $5-15 \mathrm{~cm}$ soil layers (Tables 3 and 4). In $0-5 \mathrm{~cm}$ layer of $\mathrm{CT}$ system, $\mathrm{T}_{2}, \mathrm{~T}_{3}$ and $\mathrm{T}_{4}$ treatments increased TOC content from $3.84 \mathrm{~g} \cdot \mathrm{kg}^{-1}$ in control $\left(\mathrm{T}_{1}\right)$ to $4.19,4.33$ and $4.45 \mathrm{~g} \cdot \mathrm{kg}^{-1}$ without $\mathrm{CR}$, and to 
Table 2. Effect of fertilizer, FYM and crop residue treatments on soil aggregate size distribution (\%) and mean weight diameter (MWD) in 5 - $15 \mathrm{~cm}$ soil layer after 2-year of the experiment.

\begin{tabular}{|c|c|c|c|c|c|c|c|c|c|}
\hline \multirow{2}{*}{$\begin{array}{c}\text { Treatment } \\
\text { No. }\end{array}$} & \multicolumn{2}{|l|}{ Treatments } & \multicolumn{7}{|c|}{ Distribution of WSA in different sizes (\%) } \\
\hline & Soybean & Wheat & $0.11-0.25 \mathrm{~mm}$ & $0.25-0.50 \mathrm{~mm}$ & $0.5-1.0 \mathrm{~mm}$ & $1.0-2.0 \mathrm{~mm}$ & $>2.0 \mathrm{Mm}$ & Total WSA (\%) & $\begin{array}{l}\text { MWD } \\
(\mathrm{mm}) \\
\end{array}$ \\
\hline \multicolumn{10}{|c|}{ Conventional tillage } \\
\hline $\mathrm{T}_{1}$ & $\mathrm{~N}_{0} \mathrm{P}_{0} \mathrm{WR}_{0}^{\dagger}$ & $\mathrm{N}_{0} \mathrm{P}_{0} \mathrm{SR}_{0}^{\ddagger}$ & 23.4 & 19.2 & 9.0 & 7.9 & 3.0 & 62.4 & 0.41 \\
\hline $\mathrm{T}_{2}$ & $\mathrm{~N}_{20} \mathrm{P}_{60} \mathrm{WR}_{0}$ & $\mathrm{~N}_{120} \mathrm{P}_{60} \mathrm{SR}_{0}$ & 21.7 & 20.4 & 10.5 & 8.6 & 3.2 & 64.4 & 0.43 \\
\hline $\mathrm{T}_{3}^{\S}$ & $\mathrm{N}_{25} \mathrm{P}_{75} \mathrm{WR}_{0}$ & $\mathrm{~N}_{150} \mathrm{P}_{75} \mathrm{SR}_{0}$ & - & - & - & - & - & - & - \\
\hline $\mathrm{T}_{4}$ & $\mathrm{~N}_{20} \mathrm{P}_{60} \mathrm{WR}_{0}+\mathrm{FYM}_{10} \hat{}$ & $\mathrm{N}_{120} \mathrm{P}_{60} \mathrm{SR}_{0}$ & 20.6 & 21.9 & 14.9 & 9.7 & 4.6 & 71.7 & 0.48 \\
\hline $\mathrm{T}_{5}$ & $\mathrm{~N}_{0} \mathrm{P}_{0} \mathrm{WR}_{6}$ & $\mathrm{~N}_{0} \mathrm{P}_{0} \mathrm{SR}_{3}$ & 22.2 & 21.0 & 15.7 & 9.2 & 4.1 & 72.1 & 0.46 \\
\hline $\mathrm{T}_{6}$ & $\mathrm{~N}_{20} \mathrm{P}_{60} \mathrm{WR}_{6}$ & $\mathrm{~N}_{120} \mathrm{P}_{60} \mathrm{SR}_{3}$ & 23.6 & 22.3 & 15.3 & 11.1 & 4.5 & 76.8 & 0.47 \\
\hline $\mathrm{T}_{7}^{\S}$ & $\mathrm{N}_{25} \mathrm{P}_{75} \mathrm{WR}_{6}$ & $\mathrm{~N}_{150} \mathrm{P}_{75} \mathrm{SR}_{3}$ & - & - & - & - & - & - & - \\
\hline $\mathrm{T}_{8}$ & $\mathrm{~N}_{20} \mathrm{P}_{60} \mathrm{WR}_{6}+\mathrm{FYM}_{10}$ & $\mathrm{~N}_{120} \mathrm{P}_{60} \mathrm{SR}_{3}$ & 20.7 & 23.2 & 15.8 & 12.1 & 5.5 & 77.3 & 0.51 \\
\hline & CT Mean & & 22.0 & 21.3 & 13.5 & 9.8 & 4.2 & 70.8 & 0.46 \\
\hline & $L S D(0.05)$ & & ns & ns & 2.2 & 1.4 & 0.57 & 1.6 & 0.01 \\
\hline \multicolumn{10}{|c|}{ Conservation agriculture } \\
\hline $\mathrm{T}_{9}$ & $\mathrm{~N}_{0} \mathrm{P}_{0} \mathrm{WR}_{0}$ & $\mathrm{~N}_{0} \mathrm{P}_{0} \mathrm{SR}_{0}$ & 22.0 & 21.3 & 10.7 & 8.2 & 3.4 & 65.6 & 0.43 \\
\hline $\mathrm{T}_{10}$ & $\mathrm{~N}_{20} \mathrm{P}_{60} \mathrm{WR}_{0}$ & $\mathrm{~N}_{120} \mathrm{P}_{60} \mathrm{SR}_{0}$ & 21.0 & 24.0 & 10.6 & 8.2 & 4.1 & 68.0 & 0.44 \\
\hline $\mathrm{T}_{11}^{\S}$ & $\mathrm{N}_{25} \mathrm{P}_{75} \mathrm{WR}_{0}$ & $\mathrm{~N}_{150} \mathrm{P}_{75} \mathrm{SR}_{0}$ & - & - & - & - & - & - & - \\
\hline $\mathrm{T}_{12}$ & $\mathrm{~N}_{20} \mathrm{P}_{60} \mathrm{WR}_{0}+\mathrm{FYM}_{10}$ & $\mathrm{~N}_{120} \mathrm{P}_{60} \mathrm{SR}_{0}$ & 22.6 & 22.3 & 15.4 & 10.3 & 5.1 & 75.7 & 0.48 \\
\hline $\mathrm{T}_{13}$ & $\mathrm{~N}_{0} \mathrm{P}_{0} \mathrm{WR}_{6}$ & $\mathrm{~N}_{0} \mathrm{P}_{0} \mathrm{SR}_{3}$ & 22.5 & 21.4 & 14.5 & 9.8 & 4.3 & 72.5 & 0.46 \\
\hline $\mathrm{T}_{14}$ & $\mathrm{~N}_{20} \mathrm{P}_{60} \mathrm{WR}_{6}$ & $\mathrm{~N}_{120} \mathrm{P}_{60} \mathrm{SR}_{3}$ & 23.8 & 23.9 & 14.5 & 10.9 & 5.1 & 78.1 & 0.47 \\
\hline $\mathrm{T}_{15}^{\S}$ & $\mathrm{N}_{25} \mathrm{P}_{75} \mathrm{WR}_{6}$ & $\mathrm{~N}_{150} \mathrm{P}_{75} \mathrm{SR}_{3}$ & - & - & - & - & - & - & - \\
\hline $\mathrm{T}_{16}$ & $\mathrm{~N}_{20} \mathrm{P}_{60} \mathrm{WR}_{6}+\mathrm{FYM}_{10}$ & $\mathrm{~N}_{120} \mathrm{P}_{60} \mathrm{SR}_{3}$ & 24.6 & 24.4 & 15.5 & 11.0 & 5.3 & 80.8 & 0.47 \\
\hline & CA Mean & & 22.8 & 22.9 & 13.5 & 9.7 & 4.6 & 73.5 & 0.46 \\
\hline & LSD (0.05) & & ns & 2.2 & 1.0 & 0.83 & 0.62 & 1.2 & 0.01 \\
\hline
\end{tabular}

${ }^{\dagger} \mathrm{N}=$ fertilizer $\mathrm{N}\left(\mathrm{kg} \mathrm{N} \mathrm{ha}^{-1}\right) ; \mathrm{P}=$ fertilizer $\mathrm{P}\left(\mathrm{kg} \mathrm{P}_{2} \mathrm{O}_{5} \mathrm{ha}^{-1}\right)$; WR $=$ Wheat crop residue $\left(\mathrm{t} \cdot \mathrm{ha}^{-1}\right) ;{ }^{\ddagger} \mathrm{SR}=$ Soybean crop residue $\left(\mathrm{t} \cdot \mathrm{ha}{ }^{-1}\right) ;{ }^{\S} \mathrm{WSA}$ and $\mathrm{MWD}$ were not measured in Treatments $\left(\mathrm{T}_{3}, \mathrm{~T}_{7}, \mathrm{~T}_{11}\right.$, and $\left.\mathrm{T}_{15}\right) ; \mathrm{FYM}=$ farmyard manure $\left(\mathrm{t} \cdot \mathrm{ha}^{-1}\right) ; \mathrm{ns}=$ non-significant.

4.40, 4.83 and $5.79 \mathrm{~g} \cdot \mathrm{kg}^{-1}$ with $\mathrm{CR}\left(\mathrm{T}_{6}, \mathrm{~T}_{7}\right.$ and $\left.\mathrm{T}_{8}\right)$ after 2 years. The corresponding values of TOC content under CA system were $4.55 \mathrm{~g} \cdot \mathrm{kg}^{-1}$ in control to $4.73,4.79$ and $5.02 \mathrm{~g} \cdot \mathrm{kg}^{-1}$ without CR and to $4.95,5.07$ and $5.30 \mathrm{~g} \cdot \mathrm{kg}^{-1}$ with CR. After 4 years of these treatments, there was further improvement in TOC content from $1 \%$ to $26 \%$ in $\mathrm{CT}$ and none to $19 \%$ in CA treatments. In $5-15 \mathrm{~cm}$ layer, the increasing trends due to the application of $\mathrm{N}$ and $\mathrm{P}$ fertilizers, FYM and CR were similar to those observed in $0-5 \mathrm{~cm}$ layer, however, the magnitude was relatively lower (Table 4). The impact of applied fertilizer, FYM and CR in improving TOC content was more in $0-5 \mathrm{~cm}$ soil layer than in $5-15 \mathrm{~cm}$ soil layer under both CA and $\mathrm{CT}$ system. With the application of inorganic and organic fertilizer treatments, TOC stock significantly differed under $\mathrm{CT}$ and $\mathrm{CA}$ system with different treatments in both $0-5 \mathrm{~cm}$ and $5-15 \mathrm{~cm}$ soil layers (Tables 3 and 4). In $0-5 \mathrm{~cm}$ soil layer of CT system, $\mathrm{T}_{2}, \mathrm{~T}_{3}$ and $\mathrm{T}_{4}$ treatments increased TOC stock from $2.92 \mathrm{t} \cdot \mathrm{C} \cdot \mathrm{ha}^{-1}$ in control $\left(\mathrm{T}_{1}\right)$ to $3.19,3.29$ and $3.38 \mathrm{t} \cdot \mathrm{C} \cdot \mathrm{ha}{ }^{-1}$ without $\mathrm{CR}$, and to 3.35, 3.67 and $4.40 \mathrm{t} \cdot \mathrm{C} \cdot \mathrm{ha}^{-1}$ with $\mathrm{CR}\left(\mathrm{T}_{6}, \mathrm{~T}_{7}\right.$ and $\left.\mathrm{T}_{8}\right)$ after 2 years (Table 3). The corresponding increase of TOC stock under CA system was from $3.46 \mathrm{t} \cdot \mathrm{C} \cdot \mathrm{ha}^{-1}$ in control to $3.59,3.64$ and $3.82 \mathrm{t} \cdot \mathrm{C} \cdot \mathrm{ha}^{-1}$ without $\mathrm{CR}$ and to 3.76 , 3.85 and $4.03 \mathrm{t} \cdot \mathrm{C} \cdot \mathrm{ha}^{-1}$ with CR. In $5-15 \mathrm{~cm}$ layer, the increasing trends in TOC stocks due to the application of $\mathrm{N}$ and $\mathrm{P}$ fertilizers, FYM and CR were similar to those in $0-5 \mathrm{~cm}$ layer, however, the magnitude was relatively higher in $5-15 \mathrm{~cm}$ soil layer, obviously due to high soil mass (Table 4). After 2 years, the TN content ranged 
Table 3. Total organic C (TOC), total N (TN) and C/N ratio in 0 - $5 \mathrm{~cm}$ soil layer after 2 and 4 years of soybean-wheat rotation as influenced by fertilizers, FYM and crop residue management practices under conventional tillage (CT) and conservation agriculture (CA).

\begin{tabular}{|c|c|c|c|c|c|c|c|c|c|c|}
\hline \multirow{2}{*}{$\begin{array}{c}\text { Treatment } \\
\text { No. }\end{array}$} & \multicolumn{2}{|c|}{ Treatments } & \multicolumn{2}{|c|}{ TOC $\left(\mathrm{g} \cdot \mathrm{kg}^{-1}\right)$} & \multicolumn{2}{|c|}{ TOC $\left(\mathrm{t} \cdot \mathrm{ha}^{-1}\right)$} & \multicolumn{2}{|c|}{$\mathrm{TN}\left(\mathrm{mg} \cdot \mathrm{kg}^{-1}\right)$} & \multicolumn{2}{|c|}{$\mathrm{C} / \mathrm{N}$ ratio } \\
\hline & Soybean & Wheat & $2-y r$ & $4-y r$ & $2-y r$ & $4-y r$ & $2-y r$ & $4-y r$ & $2-\mathrm{yr}$ & $4-y r$ \\
\hline & Conventional tillage & & & & & & & & & \\
\hline $\mathrm{T}_{1}$ & $\mathrm{~N}_{0} \mathrm{P}_{0} \mathrm{WR}_{0}^{\dagger}$ & $\mathrm{N}_{0} \mathrm{P}_{0} \mathrm{SR}_{0}^{\ddagger}$ & 3.84 & 3.89 & 2.92 & 2.95 & 437 & 439 & 8.8 & 8.9 \\
\hline $\mathrm{T}_{2}$ & $\mathrm{~N}_{20} \mathrm{P}_{60} \mathrm{WR}_{0}$ & $\mathrm{~N}_{120} \mathrm{P}_{60} \mathrm{SR}_{0}$ & 4.19 & 4.31 & 3.19 & 3.28 & 514 & 518 & 8.2 & 8.3 \\
\hline $\mathrm{T}_{3}$ & $\mathrm{~N}_{25} \mathrm{P}_{75} \mathrm{WR}_{0}$ & $\mathrm{~N}_{150} \mathrm{P}_{75} \mathrm{SR}_{0}$ & 4.33 & 4.38 & 3.29 & 3.33 & 548 & 550 & 7.9 & 8.0 \\
\hline $\mathrm{T}_{4}$ & $\mathrm{~N}_{20} \mathrm{P}_{60} \mathrm{WR}_{0}+\mathrm{FYM}_{10} \hat{}$ & $\mathrm{N}_{120} \mathrm{P}_{60} \mathrm{SR}_{0}$ & 4.45 & 5.60 & 3.38 & 4.26 & 583 & 585 & 7.6 & 9.6 \\
\hline $\mathrm{T}_{5}$ & $\mathrm{~N}_{0} \mathrm{P}_{0} \mathrm{WR}_{6}$ & $\mathrm{~N}_{0} \mathrm{P}_{0} \mathrm{SR}_{3}$ & 4.02 & 4.46 & 3.05 & 3.39 & 464 & 470 & 8.7 & 9.5 \\
\hline $\mathrm{T}_{6}$ & $\mathrm{~N}_{20} \mathrm{P}_{60} \mathrm{WR}_{6}$ & $\mathrm{~N}_{120} \mathrm{P}_{60} \mathrm{SR}_{3}$ & 4.40 & 4.95 & 3.35 & 3.76 & 587 & 592 & 7.5 & 8.4 \\
\hline $\mathrm{T}_{7}$ & $\mathrm{~N}_{25} \mathrm{P}_{75} \mathrm{WR}_{6}$ & $\mathrm{~N}_{150} \mathrm{P}_{75} \mathrm{SR}_{3}$ & 4.83 & 5.35 & 3.67 & 4.07 & 655 & 658 & 7.4 & 8.1 \\
\hline \multirow[t]{2}{*}{$\mathrm{T}_{8}$} & $\mathrm{~N}_{20} \mathrm{P}_{60} \mathrm{WR}_{6}+\mathrm{FYM}_{10}$ & $\mathrm{~N}_{120} \mathrm{P}_{60} \mathrm{SR}_{3}$ & 5.79 & 5.89 & 4.40 & 4.48 & 844 & 847 & 6.9 & 7.0 \\
\hline & CT Mean & & 4.48 & 4.86 & 3.41 & 3.69 & 579 & 582 & 7.9 & 8.5 \\
\hline \multicolumn{11}{|c|}{ Conservation agriculture } \\
\hline $\mathrm{T}_{9}$ & $\mathrm{~N}_{0} \mathrm{P}_{0} \mathrm{WR}_{0}$ & $\mathrm{~N}_{0} \mathrm{P}_{0} \mathrm{SR}_{0}$ & 4.55 & 4.50 & 3.46 & 3.42 & 476 & 483 & 9.6 & 9.3 \\
\hline $\mathrm{T}_{10}$ & $\mathrm{~N}_{20} \mathrm{P}_{60} \mathrm{WR}_{0}$ & $\mathrm{~N}_{120} \mathrm{P}_{60} \mathrm{SR}_{0}$ & 4.73 & 4.75 & 3.59 & 3.61 & 542 & 547 & 8.7 & 8.7 \\
\hline $\mathrm{T}_{11}$ & $\mathrm{~N}_{25} \mathrm{P}_{75} \mathrm{WR}_{0}$ & $\mathrm{~N}_{150} \mathrm{P}_{75} \mathrm{SR}_{0}$ & 4.79 & 5.03 & 3.64 & 3.82 & 575 & 579 & 8.3 & 8.7 \\
\hline $\mathrm{T}_{12}$ & $\mathrm{~N}_{20} \mathrm{P}_{60} \mathrm{WR}_{0}+\mathrm{FYM}_{10}$ & $\mathrm{~N}_{120} \mathrm{P}_{60} \mathrm{SR}_{0}$ & 5.02 & 5.98 & 3.82 & 4.54 & 618 & 624 & 8.1 & 9.6 \\
\hline $\mathrm{T}_{13}$ & $\mathrm{~N}_{0} \mathrm{P}_{0} \mathrm{WR}_{6}$ & $\mathrm{~N}_{0} \mathrm{P}_{0} \mathrm{SR}_{3}$ & 4.90 & 4.92 & 3.73 & 3.74 & 522 & 541 & 9.4 & 9.1 \\
\hline $\mathrm{T}_{14}$ & $\mathrm{~N}_{20} \mathrm{P}_{60} \mathrm{WR}_{6}$ & $\mathrm{~N}_{120} \mathrm{P}_{60} \mathrm{SR}_{3}$ & 4.95 & 5.07 & 3.76 & 3.85 & 642 & 648 & 7.7 & 7.8 \\
\hline $\mathrm{T}_{15}$ & $\mathrm{~N}_{25} \mathrm{P}_{75} \mathrm{WR}_{6}$ & $\mathrm{~N}_{150} \mathrm{P}_{75} \mathrm{SR}_{3}$ & 5.07 & 5.65 & 3.85 & 4.29 & 683 & 692 & 7.4 & 8.2 \\
\hline \multirow[t]{7}{*}{$\mathrm{T}_{16}$} & $\mathrm{~N}_{20} \mathrm{P}_{60} \mathrm{WR}_{6}+\mathrm{FYM}_{10}$ & $\mathrm{~N}_{120} \mathrm{P}_{60} \mathrm{SR}_{3}$ & 5.30 & 5.99 & 4.03 & 4.56 & 887 & 906 & 6.0 & 6.6 \\
\hline & CA Mean & & 4.91 & 5.24 & 3.73 & 3.98 & 618 & 628 & 8.2 & 8.5 \\
\hline & Overall mean & & 4.70 & 5.05 & 3.57 & 3.83 & 599 & 605 & 8.0 & 8.5 \\
\hline & LSD (0.05) & & & & & & & & & \\
\hline & Treatment & & 0.23 & 0.52 & 0.16 & 0.39 & 13 & 15 & - & - \\
\hline & Tillage & & 0.02 & ns & 0.02 & $\mathrm{~ns}$ & 25 & 20 & - & - \\
\hline & Crop residue & & 0.15 & 0.26 & 0.11 & 0.20 & 13 & 16 & - & - \\
\hline
\end{tabular}

${ }^{\dagger} \mathrm{N}=$ fertilizer $\mathrm{N}\left(\mathrm{kg} \mathrm{N} \mathrm{ha}^{-1}\right) ; \mathrm{P}=$ fertilizer $\mathrm{P}\left(\mathrm{kg} \mathrm{P}_{2} \mathrm{O}_{5} \mathrm{ha}^{-1}\right) ; \mathrm{WR}=$ Wheat crop residue $\left(\mathrm{t} \cdot \mathrm{ha}^{-1}\right) ;{ }^{\dagger} \mathrm{SR}=$ Soybean crop residue $\left(\mathrm{t} \cdot \mathrm{ha}{ }^{-1}\right) ;{ }^{\wedge} \mathrm{FYM}=$ farmyard manure $\left(\mathrm{t} \cdot \mathrm{ha}^{-1}\right) ; \mathrm{ns}=$ non-significant.

from 437 to $844 \mathrm{mg} \cdot \mathrm{kg}^{-1}$ and 476 to $887 \mathrm{mg} \cdot \mathrm{kg}^{-1}$ in $0-5$ $\mathrm{cm}$ soil layer under $\mathrm{CT}$ and $\mathrm{CA}$ system, respectively (Table 3). The further increase in TN after 4 years was negligible. In 0 - $5 \mathrm{~cm}$ soil layer, the $\mathrm{C} / \mathrm{N}$ ratio was 8.8 in control, which became narrower with application of $\mathrm{N}$ and $\mathrm{P}, \mathrm{FYM}$ and $\mathrm{CR}$ treatments and narrowest $\mathrm{C} / \mathrm{N}$ ratio of 6.9 was found in $\mathrm{T}_{8}$ treatment under $\mathrm{CT}$ system after 2 cycles. Similarly, in CA system, the $\mathrm{C} / \mathrm{N}$ ratio was 9.6 in control that reduced to 6.0 in $\mathrm{T}_{16}$ treatment. The overall TOC stock increased over control by $10 \%, 16 \%$ and $23 \%$ without CR, and by $19 \%, 28 \%$ and $41 \%$ with $\mathrm{CR}$ in 0 $15 \mathrm{~cm}$ soil layer of CT system. The corresponding increase in TOC stock under CA system over control was by $4.7 \%, 8.4 \%$ and $12.2 \%$ without $\mathrm{CR}$ and by $12.7 \%$, $15.3 \%$ and $19.4 \%$ with CR. Of the TOC stocks found in different treatments in $0-15 \mathrm{~cm}$ surface layer of both CT and $\mathrm{CA}, 39 \%$ to $44 \%$ were present in surface $5 \mathrm{~cm}$ and only $56 \%$ to $61 \%$ in next $10 \mathrm{~cm}$ depth suggesting decrease in TOC stocks with soil depth. The treatments $\mathrm{T}_{8}$ and $\mathrm{T}_{16}$ in subsurface layer after 2 cycles leads to $41 \%$ 
Table 4. Total organic C (TOC), total N (TN) and C/N ratio in 5 - $15 \mathrm{~cm}$ soil layer after 2 and 4 years of soybean-wheat rotation as influenced by fertilizers, FYM and crop residue management practices under conventional tillage (CT) and conservation agriculture (CA).

\begin{tabular}{|c|c|c|c|c|c|c|c|c|c|c|}
\hline \multirow{2}{*}{$\begin{array}{c}\text { Treatment } \\
\text { No. }\end{array}$} & \multicolumn{2}{|c|}{ Treatments } & \multicolumn{2}{|c|}{ TOC $\left(g \cdot \mathrm{kg}^{-1}\right)$} & \multicolumn{2}{|c|}{$\operatorname{TOC}\left(\mathrm{t} \cdot \mathrm{ha}^{-1}\right)$} & \multicolumn{2}{|c|}{$\mathrm{TN}\left(\mathrm{mg} \cdot \mathrm{kg}^{-1}\right)$} & \multicolumn{2}{|c|}{$\mathrm{C} / \mathrm{N}$ ratio } \\
\hline & Soybean & Wheat & $2-y r$ & $4-y r$ & $2-y r$ & $4-y r$ & $2-y r$ & $4-y r$ & $2-y r$ & $4-y r$ \\
\hline \multicolumn{11}{|c|}{ Conventional tillage } \\
\hline $\mathrm{T}_{1}$ & $\mathrm{~N}_{0} \mathrm{P}_{0} \mathrm{WR}_{0}^{\dagger}$ & $\mathrm{N}_{0} \mathrm{P}_{0} \mathrm{SR}_{0}^{\ddagger}$ & 2.69 & 2.75 & 4.12 & 4.21 & 421 & 426 & 6.4 & 6.5 \\
\hline $\mathrm{T}_{2}$ & $\mathrm{~N}_{20} \mathrm{P}_{60} \mathrm{WR}_{0}$ & $\mathrm{~N}_{120} \mathrm{P}_{60} \mathrm{SR}_{0}$ & 2.99 & 2.99 & 4.57 & 4.58 & 478 & 487 & 6.3 & 6.1 \\
\hline $\mathrm{T}_{3}$ & $\mathrm{~N}_{25} \mathrm{P}_{75} \mathrm{WR}_{0}$ & $\mathrm{~N}_{150} \mathrm{P}_{75} \mathrm{SR}_{0}$ & 3.18 & 3.53 & 4.86 & 5.41 & 533 & 545 & 6.0 & 6.5 \\
\hline $\mathrm{T}_{4}$ & $\mathrm{~N}_{20} \mathrm{P}_{60} \mathrm{WR}_{0}+\mathrm{FYM}_{10} \hat{~}$ & $\mathrm{~N}_{120} \mathrm{P}_{60} \mathrm{SR}_{0}$ & 3.47 & 3.70 & 5.30 & 5.66 & 583 & 601 & 5.9 & 6.2 \\
\hline $\mathrm{T}_{5}$ & $\mathrm{~N}_{0} \mathrm{P}_{0} \mathrm{WR}_{6}$ & $\mathrm{~N}_{0} \mathrm{P}_{0} \mathrm{SR}_{3}$ & 2.98 & 3.43 & 4.55 & 5.24 & 477 & 484 & 6.2 & 7.1 \\
\hline $\mathrm{T}_{6}$ & $\mathrm{~N}_{20} \mathrm{P}_{60} \mathrm{WR}_{6}$ & $\mathrm{~N}_{120} \mathrm{P}_{60} \mathrm{SR}_{3}$ & 3.29 & 3.55 & 5.03 & 5.43 & 579 & 580 & 5.7 & 6.1 \\
\hline $\mathrm{T}_{7}$ & $\mathrm{~N}_{25} \mathrm{P}_{75} \mathrm{WR}_{6}$ & $\mathrm{~N}_{150} \mathrm{P}_{75} \mathrm{SR}_{3}$ & 3.48 & 4.08 & 5.32 & 6.23 & 639 & 647 & 5.4 & 6.3 \\
\hline \multirow[t]{3}{*}{$\mathrm{T}_{8}$} & $\mathrm{~N}_{20} \mathrm{P}_{60} \mathrm{WR}_{6}+\mathrm{FYM}_{10}$ & $\mathrm{~N}_{120} \mathrm{P}_{60} \mathrm{SR}_{3}$ & 3.61 & 4.18 & 5.53 & 6.39 & 822 & 834 & 4.4 & 5.0 \\
\hline & CT Mean & & 3.21 & 3.53 & 4.91 & 5.39 & 567 & 576 & 5.8 & 6.2 \\
\hline & \multicolumn{10}{|c|}{ Conservation agriculture } \\
\hline $\mathrm{T}_{9}$ & $\mathrm{~N}_{0} \mathrm{P}_{0} \mathrm{WR}_{0}$ & $\mathrm{~N}_{0} \mathrm{P}_{0} \mathrm{SR}_{0}$ & 3.10 & 3.21 & 4.75 & 4.91 & 423 & 430 & 7.3 & 7.5 \\
\hline $\mathrm{T}_{10}$ & $\mathrm{~N}_{20} \mathrm{P}_{60} \mathrm{WR}_{0}$ & $\mathrm{~N}_{120} \mathrm{P}_{60} \mathrm{SR}_{0}$ & 3.27 & 3.40 & 5.00 & 5.20 & 481 & 487 & 6.8 & 7.0 \\
\hline $\mathrm{T}_{11}$ & $\mathrm{~N}_{25} \mathrm{P}_{75} \mathrm{WR}_{0}$ & $\mathrm{~N}_{150} \mathrm{P}_{75} \mathrm{SR}_{0}$ & 3.44 & 3.48 & 5.26 & 5.32 & 547 & 561 & 6.3 & 6.2 \\
\hline $\mathrm{T}_{12}$ & $\mathrm{~N}_{20} \mathrm{P}_{60} \mathrm{WR}_{0}+\mathrm{FYM}_{10}$ & $\mathrm{~N}_{120} \mathrm{P}_{60} \mathrm{SR}_{0}$ & 3.53 & 3.73 & 5.40 & 5.70 & 566 & 570 & 6.2 & 6.5 \\
\hline $\mathrm{T}_{13}$ & $\mathrm{~N}_{0} \mathrm{P}_{0} \mathrm{WR}_{6}$ & $\mathrm{~N}_{0} \mathrm{P}_{0} \mathrm{SR}_{3}$ & 3.31 & 3.50 & 5.06 & 5.36 & 469 & 472 & 7.1 & 7.4 \\
\hline $\mathrm{T}_{14}$ & $\mathrm{~N}_{20} \mathrm{P}_{60} \mathrm{WR}_{6}$ & $\mathrm{~N}_{120} \mathrm{P}_{60} \mathrm{SR}_{3}$ & 3.59 & 3.83 & 5.49 & 5.85 & 606 & 610 & 5.9 & 6.3 \\
\hline $\mathrm{T}_{15}$ & $\mathrm{~N}_{25} \mathrm{P}_{75} \mathrm{WR}_{6}$ & $\mathrm{~N}_{150} \mathrm{P}_{75} \mathrm{SR}_{3}$ & 3.67 & 4.00 & 5.62 & 6.12 & 634 & 653 & 5.8 & 6.1 \\
\hline \multirow[t]{7}{*}{$\mathrm{T}_{16}$} & $\mathrm{~N}_{20} \mathrm{P}_{60} \mathrm{WR}_{6}+\mathrm{FYM}_{10}$ & $\mathrm{~N}_{120} \mathrm{P}_{60} \mathrm{SR}_{3}$ & 3.77 & 4.44 & 5.77 & 6.79 & 678 & 687 & 5.6 & 6.5 \\
\hline & CA Mean & & 3.46 & 3.70 & 5.29 & 5.66 & 551 & 559 & 6.4 & 6.7 \\
\hline & Overall mean & & 3.34 & 3.61 & 5.10 & 5.52 & 559 & 567 & 6.1 & 6.5 \\
\hline & \multicolumn{10}{|l|}{ LSD (0.05) } \\
\hline & Treatment & & 0.20 & 0.37 & 0.29 & 0.56 & 11.7 & 14 & - & - \\
\hline & Tillage & & ns & $\mathrm{ns}$ & $\mathrm{ns}$ & $\mathrm{ns}$ & 12.5 & ns & - & - \\
\hline & Crop residue & & 0.15 & ns & 0.23 & $\mathrm{~ns}$ & 15.2 & 12 & - & - \\
\hline
\end{tabular}

${ }^{\dagger} \mathrm{N}=$ fertilizer $\mathrm{N}\left(\mathrm{kg} \mathrm{N} \mathrm{ha}^{-1}\right) ; \mathrm{P}=$ fertilizer $\mathrm{P}\left(\mathrm{kg} \mathrm{P}_{2} \mathrm{O}_{5} \mathrm{ha}^{-1}\right) ; \mathrm{WR}=$ Wheat crop residue $\left(\mathrm{t} \cdot \mathrm{ha}^{-1}\right) ;{ }^{\dagger} \mathrm{SR}=$ Soybean crop residue $\left(\mathrm{t} \cdot \mathrm{ha}{ }^{-1}\right) ;{ }^{\wedge} \mathrm{FYM}=$ farmyard manure $\left(\mathrm{t} \cdot \mathrm{ha}^{-1}\right) ; \mathrm{ns}=$ non-significant.

and 39\% higher TOC stocks over CT-control in CT and $\mathrm{CA}$, respectively. The corresponding changes after $4 \mathrm{cy}-$ cles of the experiment in TOC stocks were $52 \%$ and $59 \%$.

\subsubsection{Water Soluble C}

After 2 years, in $0-5 \mathrm{~cm}$ soil layer of CT system, $\mathrm{T}_{2}, \mathrm{~T}_{3}$ and $\mathrm{T}_{4}$ treatments increased WSC content from 11 $\mathrm{mg} \cdot \mathrm{kg}^{-1}$ in control $\left(\mathrm{T}_{1}\right)$ to 16,19 and $23 \mathrm{mg} \mathrm{kg}^{-1}$ without $\mathrm{CR}$, and to 20,21 and $28 \mathrm{mg} \cdot \mathrm{kg}^{-1}$ with $\mathrm{CR}\left(\mathrm{T}_{6}, \mathrm{~T}_{7}\right.$ and $\mathrm{T}_{8}$ ), respectively (Table 5 ). The corresponding increase of WSC content under CA system was from $20 \mathrm{mg} \cdot \mathrm{kg}^{-1}$ in control to 25,26 and $27 \mathrm{mg} \cdot \mathrm{kg}^{-1}$ without $\mathrm{CR}$ and to 26 , 27 and $29 \mathrm{mg} \cdot \mathrm{kg}^{-1}$ with CR. The trends were similar after 4 years indicating a small improvement in WSC content of different treatments. Similar increasing trends were observed in $5-15 \mathrm{~cm}$ soil layer, however, the magnitude was relatively lower (Table 6).

\subsubsection{Particulate Organic $\mathrm{C}$ and $\mathrm{N}$}

After 2 years of the experiment, in $0-5 \mathrm{~cm}$ soil layer of CT system, $T_{2}, T_{3}$ and $T_{4}$ treatments increased POC content from $390 \mathrm{mg} \cdot \mathrm{kg}^{-1}$ in control $\left(\mathrm{T}_{1}\right)$ to 550,646 and $780 \mathrm{mg} \cdot \mathrm{kg}^{-1}$ without $\mathrm{CR}$, and to 920,1040 and 1310 $\mathrm{mg} \cdot \mathrm{kg}^{-1}$ with $\mathrm{CR}\left(\mathrm{T}_{6}, \mathrm{~T}_{7}\right.$ and $\left.\mathrm{T}_{8}\right)$, respectively (Table 5). The corresponding increase of POC content under CA system was from $500 \mathrm{mg} \cdot \mathrm{kg}^{-1}$ in control to 690,730 and $910 \mathrm{mg} \cdot \mathrm{kg}^{-1}$ without $\mathrm{CR}$ and 1050,1110 and 1440 $\mathrm{mg} \cdot \mathrm{kg}^{-1}$ with $\mathrm{CR}$. The trends were similar after 4 cycles of soybean-wheat rotation indicating a small improvement in POC content of different treatments. In subsur- 
Table 5. Water soluble C (WSC), particulate organic C (POC), particulate organic N (PON), light fraction organic C (LFOC), and light fraction organic $N(L F O N)$ in $0-5 \mathrm{~cm}$ soil layer after 2 and 4 years of soybean-wheat rotation as influenced by fertilizers, FYM and crop residue management practices under conventional tillage (CT) and conservation agriculture (CA).

\begin{tabular}{|c|c|c|c|c|c|c|c|c|c|c|c|c|}
\hline \multirow[t]{2}{*}{ Treatment No. } & \multicolumn{2}{|c|}{ Treatments } & \multicolumn{2}{|c|}{ WSC $\left(\mathrm{mg} \cdot \mathrm{kg}^{-1}\right)$} & \multicolumn{2}{|c|}{$\mathrm{POC}\left(\mathrm{mg} \cdot \mathrm{kg}^{-1}\right)$} & \multicolumn{2}{|c|}{$\mathrm{PON}\left(\mathrm{mg} \cdot \mathrm{kg}^{-1}\right)$} & \multicolumn{2}{|c|}{ LFOC $\left(\mathrm{mg} \cdot \mathrm{kg}^{-1}\right)$} & \multicolumn{2}{|c|}{$\mathrm{LFON}\left(\mathrm{mg} \cdot \mathrm{kg}^{-1}\right)$} \\
\hline & Soybean & Wheat & 2-year & 4-year & 2-year & 4-year & 2-year & 4-year & 2-year & 4-year & 2-year & 4-year \\
\hline \multicolumn{13}{|c|}{ Conventional tillage } \\
\hline $\mathrm{T}_{1}$ & $\mathrm{~N}_{0} \mathrm{P}_{0} \mathrm{WR}_{0}^{\dagger}$ & $\mathrm{N}_{0} \mathrm{P}_{0} \mathrm{SR}_{0}^{\ddagger}$ & 11.0 & 12.4 & 390 & 409 & 17.4 & 17.6 & 40.0 & 41.1 & 5.6 & 5.8 \\
\hline $\mathrm{T}_{2}$ & $\mathrm{~N}_{20} \mathrm{P}_{60} \mathrm{WR}_{0}$ & $\mathrm{~N}_{120} \mathrm{P}_{60} \mathrm{SR}_{0}$ & 16.2 & 16.6 & 550 & 643 & 59.5 & 61.6 & 76.7 & 79.7 & 8.6 & 9.2 \\
\hline $\mathrm{T}_{3}$ & $\mathrm{~N}_{25} \mathrm{P}_{75} \mathrm{WR}_{0}$ & $\mathrm{~N}_{150} \mathrm{P}_{75} \mathrm{SR}_{0}$ & 19.0 & 20.6 & 646 & 690 & 79.9 & 80.3 & 94.2 & 94.9 & 10.0 & 11.1 \\
\hline $\mathrm{T}_{4}$ & $\mathrm{~N}_{20} \mathrm{P}_{60} \mathrm{WR}_{0}+\mathrm{FYM}_{10} \hat{}$ & $\mathrm{N}_{120} \mathrm{P}_{60} \mathrm{SR}_{0}$ & 22.9 & 24.0 & 780 & 839 & 96.6 & 99.1 & 149.1 & 149.9 & 13.0 & 14.0 \\
\hline $\mathrm{T}_{5}$ & $\mathrm{~N}_{0} \mathrm{P}_{0} \mathrm{WR}_{6}$ & $\mathrm{~N}_{0} \mathrm{P}_{0} \mathrm{SR}_{3}$ & 12.6 & 13.7 & 500 & 613 & 19.6 & 20.3 & 62.9 & 64.2 & 8.3 & 8.8 \\
\hline $\mathrm{T}_{6}$ & $\mathrm{~N}_{20} \mathrm{P}_{60} \mathrm{WR}_{6}$ & $\mathrm{~N}_{120} \mathrm{P}_{60} \mathrm{SR}_{3}$ & 20.4 & 22.1 & 920 & 964 & 69.0 & 69.2 & 93.2 & 95.5 & 9.9 & 10.3 \\
\hline $\mathrm{T}_{7}$ & $\mathrm{~N}_{25} \mathrm{P}_{75} \mathrm{WR}_{6}$ & $\mathrm{~N}_{150} \mathrm{P}_{75} \mathrm{SR}_{3}$ & 20.9 & 22.6 & 1040 & 1045 & 89.1 & 90.8 & 130.4 & 134.0 & 12.6 & 13.2 \\
\hline $\mathrm{T}_{8}$ & $\mathrm{~N}_{20} \mathrm{P}_{60} \mathrm{WR}_{6}+\mathrm{FYM}_{10}$ & $\mathrm{~N}_{120} \mathrm{P}_{60} \mathrm{SR}_{3}$ & 28.4 & 25.8 & 1310 & 1332 & 101.9 & 104.2 & 171.0 & 171.7 & 14.1 & 14.6 \\
\hline & CT Mean & & 18.9 & 19.7 & 767 & 817 & 66.6 & 67.9 & 102.2 & 103.9 & 10.3 & 10.9 \\
\hline \multicolumn{13}{|c|}{ Conservation agriculture } \\
\hline $\mathrm{T}_{9}$ & $\mathrm{~N}_{0} \mathrm{P}_{0} \mathrm{WR}_{0}$ & $\mathrm{~N}_{0} \mathrm{P}_{0} \mathrm{SR}_{0}$ & 20.1 & 20.9 & 500 & 511 & 23.5 & 23.7 & 45.9 & 48.2 & 6.2 & 6.6 \\
\hline $\mathrm{T}_{10}$ & $\mathrm{~N}_{20} \mathrm{P}_{60} \mathrm{WR}_{0}$ & $\mathrm{~N}_{120} \mathrm{P}_{60} \mathrm{SR}_{0}$ & 24.7 & 24.8 & 690 & 715 & 70.4 & 70.7 & 87.1 & 88.5 & 9.4 & 9.6 \\
\hline $\mathrm{T}_{11}$ & $\mathrm{~N}_{25} \mathrm{P}_{75} \mathrm{WR}_{0}$ & $\mathrm{~N}_{150} \mathrm{P}_{75} \mathrm{SR}_{0}$ & 26.1 & 26.4 & 730 & 807 & 89.3 & 91.3 & 121.3 & 122.7 & 12.2 & 12.7 \\
\hline $\mathrm{T}_{12}$ & $\mathrm{~N}_{20} \mathrm{P}_{60} \mathrm{WR}_{0}+\mathrm{FYM}_{10}$ & $\mathrm{~N}_{120} \mathrm{P}_{60} \mathrm{SR}_{0}$ & 27.0 & 28.2 & 910 & 942 & 112.2 & 116.1 & 174.0 & 176.4 & 13.7 & 14.0 \\
\hline $\mathrm{T}_{13}$ & $\begin{array}{lll}\mathrm{N}_{0} & \mathrm{P}_{0} & \mathrm{WR}_{6}\end{array}$ & $\mathrm{~N}_{0} \mathrm{P}_{0} \mathrm{SR}_{3}$ & 23.5 & 25.2 & 630 & 699 & 26.9 & 27.1 & 69.4 & 70.7 & 8.7 & 9.1 \\
\hline $\mathrm{T}_{14}$ & $\mathrm{~N}_{20} \mathrm{P}_{60} \mathrm{WR}_{6}$ & $\mathrm{~N}_{120} \mathrm{P}_{60} \mathrm{SR}_{3}$ & 26.2 & 28.1 & 1050 & 1164 & 80.7 & 82.5 & 105.1 & 106.5 & 10.8 & 11.5 \\
\hline $\mathrm{T}_{15}$ & $\mathrm{~N}_{25} \mathrm{P}_{75} \mathrm{WR}_{6}$ & $\mathrm{~N}_{150} \mathrm{P}_{75} \mathrm{SR}_{3}$ & 27.2 & 27.7 & 1110 & 1262 & 102.9 & 103.9 & 141.0 & 142.1 & 13.3 & 13.7 \\
\hline \multirow[t]{2}{*}{$\mathrm{T}_{16}$} & $\mathrm{~N}_{20} \mathrm{P}_{60} \mathrm{WR}_{6}+\mathrm{FYM}_{10}$ & $\mathrm{~N}_{120} \mathrm{P}_{60} \mathrm{SR}_{3}$ & 29.2 & 29.7 & 1440 & 1452 & 122.2 & 129.4 & 193.5 & 194.6 & 14.6 & 14.9 \\
\hline & CA Mean & & 25.5 & 26.4 & 882 & 944 & 78.5 & 80.6 & 117.2 & 118.7 & 11.1 & 11.5 \\
\hline & Overall mean & & 22.2 & 23.1 & 825 & 880 & 72.6 & 74.2 & 109.7 & 111.3 & 10.7 & 11.2 \\
\hline \multicolumn{13}{|c|}{$L S D(0.05)$} \\
\hline & Treatment & & 0.4 & 2.4 & 38 & 66 & 1.4 & 3.9 & 5.3 & 4.1 & 0.5 & 1.0 \\
\hline & Tillage & & 0.8 & $\mathrm{~ns}$ & 108 & ns & 4.8 & 7.0 & 12.4 & 6.7 & ns & ns \\
\hline & Crop residue & & 0.4 & 1.7 & 55 & 49 & 2.3 & 4.3 & 2.7 & 3.8 & 0.5 & 0.9 \\
\hline
\end{tabular}

${ }^{\dagger} \mathrm{N}=$ fertilizer $\mathrm{N}\left(\mathrm{kg} \mathrm{N} \mathrm{ha}^{-1}\right) ; \mathrm{P}=$ fertilizer $\mathrm{P}\left(\mathrm{kg} \mathrm{P}_{2} \mathrm{O}_{5} \mathrm{ha}^{-1}\right)$; WR $=$ Wheat crop residue $\left(\mathrm{t} \cdot \mathrm{ha}^{-1}\right) ;{ }^{\dagger} \mathrm{SR}=$ Soybean crop residue $\left(\mathrm{t} \cdot \mathrm{ha}{ }^{-1}\right) ;{ }^{\wedge} \mathrm{FYM}=$ farmyard manure $\left(\mathrm{t} \cdot \mathrm{ha}^{-1}\right) ; \mathrm{ns}=$ non-significant.

face layer, similar increasing trends were observed, however, the magnitude was relatively lower (Table 6). Results on PON content after 2-year showed that in 0-5 $\mathrm{cm}$ soil layer of CT system, $\mathrm{T}_{2}, \mathrm{~T}_{3}$ and $\mathrm{T}_{4}$ treatments increased PON content from $17 \mathrm{mg} \cdot \mathrm{kg}^{-1}$ in control $\left(\mathrm{T}_{1}\right)$ to 60,80 and $97 \mathrm{mg} \cdot \mathrm{kg}^{-1}$ without $\mathrm{CR}$, and to 69,89 and 102 $\mathrm{mg} \cdot \mathrm{kg}^{-1}$ with $\mathrm{CR}\left(\mathrm{T}_{6}, \mathrm{~T}_{7}\right.$ and $\left.\mathrm{T}_{8}\right)$ (Table 5). The corresponding increase of PON content under CA system was from $23.5 \mathrm{mg} \cdot \mathrm{kg}^{-1}$ in control to 70,89 and $112 \mathrm{mg} \cdot \mathrm{kg}^{-1}$ without $\mathrm{CR}$ and 81,103 and $122 \mathrm{mg} \cdot \mathrm{kg}^{-1}$ with CR. Small improvement in PON content was observed after 4 years of the experiment. The increasing trends in PON content were observed in subsurface layer, however, the magnitude was relatively lower (Table 6).

\subsubsection{Light Fraction Organic $\mathbf{C}$ and $\mathbf{N}$}

Results on LFOC content in 2-year experiment showed that in $0-5 \mathrm{~cm}$ soil layer of CT system, $\mathrm{T}_{2}, \mathrm{~T}_{3}$ and $\mathrm{T}_{4}$ treatments increased LFOC content from $40 \mathrm{mg} \cdot \mathrm{kg}^{-1}$ in control $\left(\mathrm{T}_{1}\right)$ to 77,94 and $149 \mathrm{mg} \cdot \mathrm{kg}^{-1}$ without $\mathrm{CR}$, and to 93,130 and $171 \mathrm{mg} \cdot \mathrm{kg}^{-1}$ with $\mathrm{CR}\left(\mathrm{T}_{6}, \mathrm{~T}_{7}\right.$ and $\left.\mathrm{T}_{8}\right)$, respectively (Table 5). The corresponding increase of 
Table 6. Water soluble C (WSC), particulate organic C (POC), particulate organic N (PON), light fraction organic C (LFOC), and light fraction organic $N$ (LFON) in 5 - $15 \mathrm{~cm}$ soil layer after 2 and 4 years of soybean-wheat rotation as influenced by fertilizers, FYM and crop residue management practices under conventional tillage (CT) and conservation agriculture (CA).

\begin{tabular}{|c|c|c|c|c|c|c|c|c|c|c|c|c|}
\hline \multirow[t]{2}{*}{ Treatment No. } & \multicolumn{2}{|c|}{ Treatments } & \multicolumn{2}{|c|}{$\mathrm{WSC}\left(\mathrm{mg} \cdot \mathrm{kg}^{-1}\right)$} & \multicolumn{2}{|c|}{$\mathrm{POC}\left(\mathrm{mg} \cdot \mathrm{kg}^{-1}\right)$} & \multicolumn{2}{|c|}{$\mathrm{PON}\left(\mathrm{mg} \cdot \mathrm{kg}^{-1}\right)$} & \multicolumn{2}{|c|}{ LFOC $\left(\mathrm{mg} \cdot \mathrm{kg}^{-1}\right)$} & \multicolumn{2}{|c|}{$\mathrm{LFON}\left(\mathrm{mg} \cdot \mathrm{kg}^{-1}\right)$} \\
\hline & Soybean & Wheat & 2-year & 4-year & 2-year & 4-year & 2-year & 4-year & 2-year & 4-year & 2-year & 4-year \\
\hline \multicolumn{13}{|c|}{ Conventional tillage } \\
\hline $\mathrm{T}_{1}$ & $\mathrm{~N}_{0} \mathrm{P}_{0} \mathrm{WR}_{0}^{\dagger}$ & $\mathrm{N}_{0} \mathrm{P}_{0} \mathrm{SR}_{0}^{\ddagger}$ & 8.7 & 10.2 & 260 & 313 & 15.8 & 16.1 & 32.3 & 32.4 & 5.1 & 5.6 \\
\hline $\mathrm{T}_{2}$ & $\mathrm{~N}_{20} \mathrm{P}_{60} \mathrm{WR}_{0}$ & $\mathrm{~N}_{120} \mathrm{P}_{60} \mathrm{SR}_{0}$ & 10.6 & 11.8 & 410 & 473 & 47.3 & 50.7 & 58.2 & 59.0 & 7.9 & 8.1 \\
\hline $\mathrm{T}_{3}$ & $\mathrm{~N}_{25} \mathrm{P}_{75} \mathrm{WR}_{0}$ & $\mathrm{~N}_{150} \mathrm{P}_{75} \mathrm{SR}_{0}$ & 12.3 & 13.8 & 520 & 537 & 67.7 & 71.5 & 79.3 & 81.0 & 10.0 & 11.5 \\
\hline $\mathrm{T}_{4}$ & $\mathrm{~N}_{20} \mathrm{P}_{60} \mathrm{WR}_{0}+\mathrm{FYM}_{10} \hat{}$ & $\mathrm{N}_{120} \mathrm{P}_{60} \mathrm{SR}_{0}$ & 14.1 & 16.8 & 600 & 687 & 85.3 & 86.4 & 128.8 & 130.0 & 11.0 & 11.4 \\
\hline $\mathrm{T}_{5}$ & $\mathrm{~N}_{0} \mathrm{P}_{0} \mathrm{WR}_{6}$ & $\mathrm{~N}_{0} \mathrm{P}_{0} \mathrm{SR}_{3}$ & 10.3 & 12.2 & 340 & 380 & 16.9 & 17.6 & 50.8 & 52.4 & 7.4 & 8.1 \\
\hline $\mathrm{T}_{6}$ & $\mathrm{~N}_{20} \mathrm{P}_{60} \mathrm{WR}_{6}$ & $\mathrm{~N}_{120} \mathrm{P}_{60} \mathrm{SR}_{3}$ & 12.9 & 13.9 & 590 & 680 & 61.3 & 67.8 & 65.8 & 66.8 & 7.1 & 8.2 \\
\hline $\mathrm{T}_{7}$ & $\mathrm{~N}_{25} \mathrm{P}_{75} \mathrm{WR}_{6}$ & $\mathrm{~N}_{150} \mathrm{P}_{75} \mathrm{SR}_{3}$ & 14.8 & 17.1 & 650 & 709 & 86.1 & 87.8 & 89.7 & 90.5 & 9.6 & 10.8 \\
\hline $\mathrm{T}_{8}$ & $\mathrm{~N}_{20} \mathrm{P}_{60} \mathrm{WR}_{6}+\mathrm{FYM}_{10}$ & $\mathrm{~N}_{120} \mathrm{P}_{60} \mathrm{SR}_{3}$ & 16.4 & 19.2 & 710 & 838 & 99.6 & 100.5 & 125.2 & 125.8 & 10.5 & 11.2 \\
\hline & CT Mean & & 12.5 & 14.4 & 510 & 577 & 60.0 & 62.3 & 78.8 & 79.7 & 8.6 & 9.4 \\
\hline \multicolumn{13}{|c|}{ Conservation agriculture } \\
\hline $\mathrm{T}_{9}$ & $\mathrm{~N}_{0} \mathrm{P}_{0} \mathrm{WR}_{0}$ & $\mathrm{~N}_{0} \mathrm{P}_{0} \mathrm{SR}_{0}$ & 10.6 & 11.2 & 320 & 367 & 20.6 & 21.3 & 37.8 & 40.1 & 5.6 & 5.9 \\
\hline $\mathrm{T}_{10}$ & $\mathrm{~N}_{20} \mathrm{P}_{60} \mathrm{WR}_{0}$ & $\mathrm{~N}_{120} \mathrm{P}_{60} \mathrm{SR}_{0}$ & 13.4 & 15.7 & 460 & 531 & 61.7 & 62.3 & 69.8 & 71.0 & 8.1 & 8.7 \\
\hline $\mathrm{T}_{11}$ & $\mathrm{~N}_{25} \mathrm{P}_{75} \mathrm{WR}_{0}$ & $\mathrm{~N}_{150} \mathrm{P}_{75} \mathrm{SR}_{0}$ & 13.7 & 19.1 & 580 & 617 & 81.0 & 82.0 & 104.0 & 105.8 & 11.9 & 13.7 \\
\hline $\mathrm{T}_{12}$ & $\mathrm{~N}_{20} \mathrm{P}_{60} \mathrm{WR}_{0}+\mathrm{FYM}_{10}$ & $\mathrm{~N}_{120} \mathrm{P}_{60} \mathrm{SR}_{0}$ & 20.2 & 21.9 & 630 & 700 & 108.0 & 109.2 & 157.9 & 159.4 & 13.0 & 13.4 \\
\hline $\mathrm{T}_{13}$ & $\mathrm{~N}_{0} \mathrm{P}_{0} \mathrm{WR}_{6}$ & $\mathrm{~N}_{0} \mathrm{P}_{0} \mathrm{SR}_{3}$ & 12.6 & 13.3 & 400 & 440 & 22.6 & 23.2 & 57.3 & 59.2 & 8.0 & 8.5 \\
\hline $\mathrm{T}_{14}$ & $\mathrm{~N}_{20} \mathrm{P}_{60} \mathrm{WR}_{6}$ & $\mathrm{~N}_{120} \mathrm{P}_{60} \mathrm{SR}_{3}$ & 15.1 & 16.5 & 660 & 683 & 74.3 & 74.7 & 84.9 & 86.1 & 9.3 & 9.8 \\
\hline $\mathrm{T}_{15}$ & $\mathrm{~N}_{25} \mathrm{P}_{75} \mathrm{WR}_{6}$ & $\mathrm{~N}_{150} \mathrm{P}_{75} \mathrm{SR}_{3}$ & 18.5 & 20.7 & 710 & 810 & 98.7 & 99.5 & 109.2 & 111.9 & 11.4 & 12.2 \\
\hline \multirow[t]{7}{*}{$\mathrm{T}_{16}$} & $\mathrm{~N}_{20} \mathrm{P}_{60} \mathrm{WR}_{6}+\mathrm{FYM}_{10}$ & $\mathrm{~N}_{120} \mathrm{P}_{60} \mathrm{SR}_{3}$ & 26.0 & 26.4 & 780 & 827 & 115.3 & 124.1 & 153.6 & 154.4 & 12.3 & 13.0 \\
\hline & CA Mean & & 16.3 & 18.1 & 567 & 622 & 72.8 & 74.6 & 96.8 & 98.5 & 10.0 & 10.7 \\
\hline & Overall mean & & 14.4 & 16.2 & 539 & 600 & 66.4 & 68.4 & 87.8 & 89.1 & 9.3 & 10.0 \\
\hline & LSD (0.05) & & & & & & & & & & & \\
\hline & Treatment & & 0.4 & 2.4 & 34 & 65 & 1.8 & 3.2 & 4.8 & 3.5 & 0.5 & 0.9 \\
\hline & Tillage & & Ns & 2.5 & $\mathrm{~ns}$ & ns & 3.1 & 3.1 & 1.3 & 3.4 & 0.8 & $\mathrm{~ns}$ \\
\hline & Crop residue & & 0.4 & $\mathrm{~ns}$ & 34 & 74 & 1.0 & 2.6 & 3.9 & 3.6 & 0.2 & $\mathrm{~ns}$ \\
\hline
\end{tabular}

${ }^{\dagger} \mathrm{N}=$ fertilizer $\mathrm{N}\left(\mathrm{kg} \mathrm{N} \mathrm{ha}^{-1}\right) ; \mathrm{P}=$ fertilizer $\mathrm{P}\left(\mathrm{kg} \mathrm{P}_{2} \mathrm{O}_{5} \mathrm{ha}^{-1}\right) ; \mathrm{WR}=$ Wheat crop residue $\left(\mathrm{t} \cdot \mathrm{ha}{ }^{-1}\right) ;{ }^{\dagger} \mathrm{SR}=\mathrm{Soybean}$ crop residue $\left(\mathrm{t} \cdot \mathrm{ha}{ }^{-1}\right) ;{ }^{\wedge} \mathrm{FYM}=$ farmyard manure $\left(\mathrm{t} \cdot \mathrm{ha}^{-1}\right) ; \mathrm{ns}=$ non-significant.

LFOC content under CA system was from $45.9 \mathrm{mg} \cdot \mathrm{kg}^{-1}$ in control to 87,121 and $174 \mathrm{mg} \cdot \mathrm{kg}^{-1}$ without $\mathrm{CR}$ and 105, 141 and $194 \mathrm{mg} \cdot \mathrm{kg}^{-1}$ with CR. After 4 years, there was a further increase in LFOC in most of the treatments. The trends were similar after 4 years of soybean-wheat experiment indicating a negligible improvement in LFOC content of different treatments. In $5-15 \mathrm{~cm}$ layer, the increasing trends in LFOC content due to the application of $\mathrm{N}$ and $\mathrm{P}$ fertilizers, FYM and CR were similar to those observed in $0-5 \mathrm{~cm}$ layer, however, the magnitude was relatively lower (Table 6).

Results on LFON content in 2-year experiment showed that in $0-5 \mathrm{~cm}$ soil layer of CT system, $\mathrm{T}_{2}, \mathrm{~T}_{3}$ and $\mathrm{T}_{4}$ treatments increased LFON content from 5.6 $\mathrm{mg} \cdot \mathrm{kg}^{-1}$ in control $\left(\mathrm{T}_{1}\right)$ to $8.6,10.0$ and $13.0 \mathrm{mg} \cdot \mathrm{kg}^{-1}$ without $\mathrm{CR}$, and to $9.9,12.6$ and $14.1 \mathrm{mg} \cdot \mathrm{kg}^{-1}$ with $\mathrm{CR}$ $\left(\mathrm{T}_{6}, \mathrm{~T}_{7}\right.$ and $\left.\mathrm{T}_{8}\right)$, respectively (Table 5$)$. The corresponding increase of LFON content under CA system was from $6.2 \mathrm{mg} \cdot \mathrm{kg}^{-1}$ in control to $9.4,12.2$ and $13.7 \mathrm{mg} \cdot \mathrm{kg}^{-1}$ without $\mathrm{CR}$ and $10.8,13.3$ and $14.6 \mathrm{mg} \cdot \mathrm{kg}^{-1}$ with $\mathrm{CR}$. After 4 years, there was a small improvement in LFON in most of the treatments. In $5-15 \mathrm{~cm}$ layer, the increasing trends in LFON content due to the application of $\mathrm{N}$ and $\mathrm{P}$ fertilizers, FYM and CR were similar to those observed in $0-5 \mathrm{~cm}$ layer, however, the magnitude was relatively lower (Table 6).

In general, the impact of applied fertilizer, FYM and CR in improving WSC, POC, PON, LFOC and LFON content was significant in $0-5 \mathrm{~cm}$ soil layer and was substantially higher than in $5-15 \mathrm{~cm}$ soil layer under 
both CA and CT system.

\subsection{Soil Biological Properties}

\subsubsection{Potentially Mineralizable $\mathbf{N}$}

Results on PMN content after 2 years of the experiment showed that in $0-5 \mathrm{~cm}$ soil layer of CT system, $\mathrm{T}_{2}, \mathrm{~T}_{3}$ and $\mathrm{T}_{4}$ treatments increased PMN content from $2.7 \mathrm{mg} \cdot \mathrm{kg}^{-1}$ $7 \mathrm{~d}^{-1}$ in control $\left(\mathrm{T}_{1}\right)$ to $2.9,3.9$ and $5.1 \mathrm{mg} \cdot \mathrm{kg}^{-1} 7 \mathrm{~d}^{-1}$ without $\mathrm{CR}$, and to $6.9,8.4$ and $9.7 \mathrm{mg} \cdot \mathrm{kg}^{-1} 7 \mathrm{~d}^{-1}$ with $\mathrm{CR}\left(\mathrm{T}_{6}\right.$, $\mathrm{T}_{7}$ and $\mathrm{T}_{8}$ ), respectively (Table 7 ). The corresponding increase of PMN content under CA system was from 3.6 $\mathrm{mg} \cdot \mathrm{kg}^{-1} 7 \mathrm{~d}^{-1}$ in control to $3.9,5.1$ and $6.5 \mathrm{mg} \cdot \mathrm{kg}^{-1} 7 \mathrm{~d}^{-1}$ without $\mathrm{CR}$ and to $8.9,10.3$ and $12.1 \mathrm{mg} \cdot \mathrm{kg}^{-1} 7 \mathrm{~d}^{-1}$ with
CR. The trends were similar after 4 years of soybeanwheat experiment indicating a small improvement in PMN content of different treatments. In $5-15 \mathrm{~cm}$ layer, the increasing trends due to the application of $\mathrm{N}$ and $\mathrm{P}$ fertilizers, FYM and CR were similar to those observed in $0-5 \mathrm{~cm}$ layer, however, the magnitude was relatively lower (Table 7).

\subsubsection{Microbial Biomass $C$ and $N$}

Results on $\mathrm{MBC}$ content after 2 years of the experiment showed that in CT system, $\mathrm{T}_{2}, \mathrm{~T}_{3}$ and $\mathrm{T}_{4}$ treatments in creased MBC content from $93 \mathrm{mg} \cdot \mathrm{kg}^{-1}$ in control $\left(\mathrm{T}_{1}\right)$ to 126,136 and $156 \mathrm{mg} \cdot \mathrm{kg}^{-1}$ without $\mathrm{CR}$, and to 147,162

Table 7. Potentially mineralizable N (PMN), microbial biomass C (MBC) and microbial biomass $N$ (MBN) in 0 - $5 \mathrm{~cm}$ soil layer after 2 and 4 years of soybean-wheat rotation as influenced by fertilizers, FYM and crop residue management practices under conventional tillage (CT) and conservation agriculture (CA).

\begin{tabular}{|c|c|c|c|c|c|c|c|c|c|c|}
\hline \multirow{3}{*}{$\begin{array}{c}\text { Treatment } \\
\text { No. }\end{array}$} & \multicolumn{2}{|c|}{ Treatments } & \multicolumn{4}{|c|}{$\mathrm{PMN}\left(\mathrm{mg} \cdot \mathrm{kg}^{-1}\right)$} & \multirow{2}{*}{\multicolumn{2}{|c|}{$\begin{array}{c}\text { MBC }\left(\mathrm{mg} \cdot \mathrm{kg}^{-1}\right) \\
0-5 \mathrm{~cm}\end{array}$}} & \multirow{2}{*}{\multicolumn{2}{|c|}{$\frac{\text { MBN }\left(\mathrm{mg} \cdot \mathrm{kg}^{-1}\right)}{0-5 \mathrm{~cm}}$}} \\
\hline & \multirow[t]{2}{*}{ Soybean } & \multirow[t]{2}{*}{ Wheat } & \multicolumn{2}{|c|}{$0-5 \mathrm{~cm}$} & \multicolumn{2}{|c|}{$5-15 \mathrm{~cm}$} & & & & \\
\hline & & & $2-y r$ & $4-y r$ & $2-y r$ & $4-y r$ & $2-y r$ & $4-y r$ & $2-y r$ & $4-y r$ \\
\hline & Conventional tillage & & & & & & & & & \\
\hline $\mathrm{T}_{1}$ & $\mathrm{~N}_{0} \mathrm{P}_{0} \mathrm{WR}_{0}^{\dagger}$ & $\mathrm{N}_{0} \mathrm{P}_{0} \mathrm{SR}_{0}^{\ddagger}$ & 2.7 & 3.3 & 1.4 & 1.6 & 93 & 94 & 5.9 & 6.3 \\
\hline $\mathrm{T}_{2}$ & $\mathrm{~N}_{20} \mathrm{P}_{60} \mathrm{WR}_{0}$ & $\mathrm{~N}_{120} \mathrm{P}_{60} \mathrm{SR}_{0}$ & 2.9 & 3.4 & 2.9 & 3.2 & 126 & 132 & 9.5 & 9.8 \\
\hline $\mathrm{T}_{3}$ & $\mathrm{~N}_{25} \mathrm{P}_{75} \mathrm{WR}_{0}$ & $\mathrm{~N}_{150} \mathrm{P}_{75} \mathrm{SR}_{0}$ & 3.9 & 4.3 & 4.2 & 4.4 & 136 & 140 & 12.7 & 13.7 \\
\hline $\mathrm{T}_{4}$ & $\mathrm{~N}_{20} \mathrm{P}_{60} \mathrm{WR}_{0}+\mathrm{FYM}_{10} \hat{}$ & $\mathrm{N}_{120} \mathrm{P}_{60} \mathrm{SR}_{0}$ & 5.1 & 5.3 & 4.9 & 5.1 & 156 & 157 & 16.0 & 16.7 \\
\hline $\mathrm{T}_{5}$ & $\mathrm{~N}_{0} \mathrm{P}_{0} \mathrm{WR}_{6}$ & $\mathrm{~N}_{0} \mathrm{P}_{0} \mathrm{SR}_{3}$ & 3.3 & 3.4 & 1.8 & 1.9 & 118 & 126 & 7.0 & 7.2 \\
\hline $\mathrm{T}_{6}$ & $\mathrm{~N}_{20} \mathrm{P}_{60} \mathrm{WR}_{6}$ & $\mathrm{~N}_{120} \mathrm{P}_{60} \mathrm{SR}_{3}$ & 6.9 & 7.1 & 5.9 & 6.2 & 147 & 149 & 12.9 & 13.2 \\
\hline $\mathrm{T}_{7}$ & $\mathrm{~N}_{25} \mathrm{P}_{75} \mathrm{WR}_{6}$ & $\mathrm{~N}_{150} \mathrm{P}_{75} \mathrm{SR}_{3}$ & 8.4 & 8.7 & 7.1 & 7.5 & 162 & 189 & 17.6 & 18.1 \\
\hline \multirow[t]{3}{*}{$\mathrm{T}_{8}$} & $\mathrm{~N}_{20} \mathrm{P}_{60} \mathrm{WR}_{6}+\mathrm{FYM}_{10}$ & $\mathrm{~N}_{120} \mathrm{P}_{60} \mathrm{SR}_{3}$ & 9.7 & 10.4 & 9.3 & 9.6 & 202 & 196 & 24.3 & 25.1 \\
\hline & CT Mean & & 5.4 & 5.7 & 4.7 & 4.9 & 143 & 148 & 13.2 & 13.7 \\
\hline & Conservation agricultu & & & & & & & & & \\
\hline $\mathrm{T}_{9}$ & $\mathrm{~N}_{0} \mathrm{P}_{0} \mathrm{WR}_{0}$ & $\mathrm{~N}_{0} \mathrm{P}_{0} \mathrm{SR}_{0}$ & 3.6 & 3.8 & 1.5 & 1.8 & 116 & 119 & 6.4 & 6.8 \\
\hline $\mathrm{T}_{10}$ & $\mathrm{~N}_{20} \mathrm{P}_{60} \mathrm{WR}_{0}$ & $\mathrm{~N}_{120} \mathrm{P}_{60} \mathrm{SR}_{0}$ & 3.9 & 4.0 & 2.3 & 2.4 & 142 & 143 & 10.4 & 10.6 \\
\hline $\mathrm{T}_{11}$ & $\mathrm{~N}_{25} \mathrm{P}_{75} \mathrm{WR}_{0}$ & $\mathrm{~N}_{150} \mathrm{P}_{75} \mathrm{SR}_{0}$ & 5.1 & 5.4 & 4.0 & 4.1 & 153 & 155 & 15.6 & 16.2 \\
\hline $\mathrm{T}_{12}$ & $\mathrm{~N}_{20} \mathrm{P}_{60} \mathrm{WR}_{0}+\mathrm{FYM}_{10}$ & $\mathrm{~N}_{120} \mathrm{P}_{60} \mathrm{SR}_{0}$ & 6.5 & 7.1 & 5.5 & 5.8 & 170 & 171 & 16.8 & 17.1 \\
\hline $\mathrm{T}_{13}$ & $\mathrm{~N}_{0} \mathrm{P}_{0} \mathrm{WR}_{6}$ & $\mathrm{~N}_{0} \mathrm{P}_{0} \mathrm{SR}_{3}$ & 4.2 & 4.8 & 2.8 & 3.1 & 139 & 140 & 7.2 & 7.9 \\
\hline $\mathrm{T}_{14}$ & $\mathrm{~N}_{20} \mathrm{P}_{60} \mathrm{WR}_{6}$ & $\mathrm{~N}_{120} \mathrm{P}_{60} \mathrm{SR}_{3}$ & 8.9 & 9.5 & 5.9 & 6.8 & 159 & 162 & 15.4 & 16.0 \\
\hline $\mathrm{T}_{15}$ & $\mathrm{~N}_{25} \mathrm{P}_{75} \mathrm{WR}_{6}$ & $\mathrm{~N}_{150} \mathrm{P}_{75} \mathrm{SR}_{3}$ & 10.3 & 11.0 & 8.4 & 8.7 & 168 & 193 & 20.5 & 22.7 \\
\hline \multirow[t]{7}{*}{$\mathrm{T}_{16}$} & $\mathrm{~N}_{20} \mathrm{P}_{60} \mathrm{WR}_{6}+\mathrm{FYM}_{10}$ & $\mathrm{~N}_{120} \mathrm{P}_{60} \mathrm{SR}_{3}$ & 12.1 & 12.9 & 10.7 & 11.4 & 227 & 215 & 27.3 & 26.1 \\
\hline & CA Mean & & 6.8 & 7.3 & 5.1 & 5.5 & 159 & 162 & 15.0 & 15.4 \\
\hline & Overall mean & & 6.1 & 6.5 & 4.9 & 5.2 & 151 & 155 & 14.1 & 14.6 \\
\hline & LSD (0.05) & & & & & & & & & \\
\hline & Treatment & & 0.6 & 0.6 & 0.4 & 0.5 & 5.5 & 6.0 & 0.5 & 0.8 \\
\hline & Tillage & & ns & ns & ns & ns & 10.9 & 10.8 & 1.1 & 0.7 \\
\hline & Crop residue & & 0.4 & 0.6 & 0.7 & 0.7 & 5.7 & 6.8 & 0.6 & 0.3 \\
\hline
\end{tabular}

${ }^{\dagger} \mathrm{N}=$ fertilizer $\mathrm{N}\left(\mathrm{kg} \mathrm{N} \mathrm{ha}^{-1}\right) ; \mathrm{P}=$ fertilizer $\mathrm{P}\left(\mathrm{kg} \mathrm{P}_{2} \mathrm{O}_{5} \mathrm{ha}^{-1}\right) ; \mathrm{WR}=$ Wheat crop residue $\left(\mathrm{t} \cdot \mathrm{ha}^{-1}\right) ;{ }^{\dagger} \mathrm{SR}=$ Soybean crop residue $\left(\mathrm{t} \cdot \mathrm{ha}^{-1}\right) ;{ }^{\wedge} \mathrm{FYM}=$ farmyard manure $\left(\mathrm{t} \cdot \mathrm{ha}^{-1}\right) ; \mathrm{ns}=$ non-significant. 
and $202 \mathrm{mg} \cdot \mathrm{kg}^{-1}$ with $\mathrm{CR}\left(\mathrm{T}_{6}, \mathrm{~T}_{7}\right.$ and $\left.\mathrm{T}_{8}\right)$, respectively (Table 7). The corresponding increase of MBC content under CA system was from $116 \mathrm{mg} \cdot \mathrm{kg}^{-1}$ in control to 142 , 153 and $170 \mathrm{mg} \cdot \mathrm{kg}^{-1}$ without CR and to 159,168 and $227 \mathrm{mg} \cdot \mathrm{kg}^{-1}$ with CR. Similar trends were observed after 4 years indicating a small improvement in MBC content of different treatments. Results on MBN content after 2 years showed that in $\mathrm{CT}$ system, $\mathrm{T}_{2}, \mathrm{~T}_{3}$ and $\mathrm{T}_{4}$ treatments increased MBN content from $5.9 \mathrm{mg} \cdot \mathrm{kg}^{-1}$ in control $\left(\mathrm{T}_{1}\right)$ to $9.5,12.7$ and $16.0 \mathrm{mg} \cdot \mathrm{kg}^{-1}$ without $\mathrm{CR}$, and to 12.9 , 17.6 and $24.3 \mathrm{mg} \cdot \mathrm{kg}^{-1}$ with $\mathrm{CR}\left(\mathrm{T}_{6}, \mathrm{~T}_{7}\right.$ and $\left.\mathrm{T}_{8}\right)$, respectively (Table 7). The corresponding increase of MBN content under CA system was from $6.4 \mathrm{mg} \cdot \mathrm{kg}^{-1}$ in control to $10.4,15.6$ and $16.8 \mathrm{mg} \cdot \mathrm{kg}^{-1}$ without $\mathrm{CR}$ and to 15.4, 20.5 and $27.3 \mathrm{mg} \cdot \mathrm{kg}^{-1}$ with CR. Maximum MBN contents were found in $\mathrm{T}_{8}$ and $\mathrm{T}_{16}$ treatments plots of both $\mathrm{CT}$ and CA systems.

\subsubsection{Proportion of Labile Organic $C$ and $N$ Fractions in Total Organic $\mathrm{C}$ and Total $\mathrm{N}$}

After 2 years of the experiment, WSC accounted for $0.29 \%$ to $0.52 \%$ and $0.44 \%$ to $0.55 \%$ of TOC in different treatments of CT and CA system, respectively in $0-5 \mathrm{~cm}$ soil layer (Table 8). While POC accounted for $10.2 \%$ to $22.6 \%$ and $11.0 \%$ to $27.2 \%$, the proportion of LFOC ranged from $1.0 \%$ to $3.3 \%$ and $1.0 \%$ to $3.6 \%$ of TOC, respectively in CT and CA system. In $5-15 \mathrm{~cm}$ layer, the trends of WSC, POC and LFOC in TOC due to the application of $\mathrm{N}$ and $\mathrm{P}$ fertilizers, FYM and CR were similar to those observed in $0-5 \mathrm{~cm}$ layer (Table 9). The proportion of $\mathrm{MBC}$ ranged from $2.4 \%$ to $3.5 \%$ under $\mathrm{CT}$ and $2.5 \%$ to $4.3 \%$ of TOC under CA system, which showed gradual increase with the application of $\mathrm{N}$ and $\mathrm{P}$, FYM and CR treatments (Table 10) and was maximum in $\mathrm{N}_{20} \mathrm{P}_{60} \mathrm{WR}_{6}+\mathrm{FYM}_{10}$ treatment under both tillage systems. After 4 years, there were minor changes in the proportion of different labile $\mathrm{C}$ fractions in TOC.

PON accounted for $4.0 \%$ to $18.2 \%$ of TN content in 0 - $5 \mathrm{~cm}$ soil layer under both CT and CA system after 2 years of experimentation (Table 8). The MBN content ranged from $1.4 \%$ to $2.9 \%$ and $1.4 \%$ to $3.1 \%$ of $\mathrm{TN}$ in $\mathrm{CT}$ and CA system, respectively. The proportion of both PMN and MBN gradually increased with the application of $\mathrm{N}$ and $\mathrm{P}, \mathrm{FYM}$ and $\mathrm{CR}$ treatments. While the proportion of PMN was highest in $T_{7}$ and $T_{15}$ treatments, MBN was highest in $\mathrm{T}_{8}$ and $\mathrm{T}_{16}$ treatment under $\mathrm{CT}$ and $\mathrm{CA}$ systems respectively after 2 years of the experiment (Table 10).

\section{Discussion}

Application of FYM enhances the soil organic C content (SOC) and has direct and indirect effects on soil properties and processes. There was a significant improvement in water stable aggregation and proportion of macro-aggregates, SOC content, labile $\mathrm{C}$ and $\mathrm{N}$ fractions such as water soluble $\mathrm{C}$, particulate and light fraction organic matter, potentially mineralizable $\mathrm{N}$ and microbial biomass with the application of $10 \mathrm{t} \mathrm{FYM} \mathrm{ha}^{-1}$ along with recommended rate of NP to soybean.

After 2 years of the experiment, total WSA in $0-5 \mathrm{~cm}$ soil layer increased from $71 \%$ in control $\left(\mathrm{T}_{1}\right)$ to a maximum of $83\left(\mathrm{~T}_{8}\right)$ and $85 \%\left(\mathrm{~T}_{16}\right)$ treatments in $\mathrm{CT}$ and CA, respectively with the addition of FYM and incorporation of crop residues (Figure 1). Likewise, the increase in total WSA was from $62 \%$ in control to 77 and $81 \%$, respectively in $5-15 \mathrm{~cm}$ layer (Figure 2), indicating the beneficial effect of integrated use of inorganic fertilizers and organic materials on the formation of aggregates over the unmanured treatments. The effects were similar after 4 years of experiment with further improvement in WSA, and the effect of tillage was statistically significant. The CA system causes less disturbance of soil and retains CR on soil surface than CT system, which could enhance and protect SOM content and improve soil structure.

TOC and TN contents of $0-5 \mathrm{~cm}$ surface soil layer significantly improved with $\mathrm{N}$ and $\mathrm{P}, \mathrm{FYM}$ and $\mathrm{CR}$ under both CT and CA systems. After 4 years of study, maximum TOC content was recorded in $\mathrm{T}_{8}$ and $\mathrm{T}_{16}$ treatments, which was 5.89 and $5.99 \mathrm{~g} \cdot \mathrm{kg}^{-1}$ in $0-5 \mathrm{~cm}$ and 4.18 and $4.44 \mathrm{~g} \cdot \mathrm{kg}^{-1}$ in $5-15 \mathrm{~cm}$ soil layer in CT and CA, respectively (Tables 3 and 4). Integrated use of inorganic fertilizers, FYM and CR significantly improved both TOC content and stock. The beneficial effects were more pronounced in $0-5 \mathrm{~cm}$ surface layer than $5-15 \mathrm{~cm}$ subsurface soil layer. Conservation tillage resulted in significantly higher TOC than $\mathrm{CT}$. The narrowest $\mathrm{C} / \mathrm{N}$ ratio of 6.9 and 6.0 was found in $0-5 \mathrm{~cm}$ in $\mathrm{N}_{20} \mathrm{P}_{60} \mathrm{WR}_{6}+\mathrm{FYM}_{10}$ $-\mathrm{N}_{120} \mathrm{P}_{60} \mathrm{SR}_{3}$ treatment of $\mathrm{CT}$ and CA system, respectively indicating improvement in $\mathrm{N}$ supplying capability of soil as the plant materials and residues in the soil decompose rapidly at narrow $\mathrm{C} / \mathrm{N}$ ratio and convert unavailable plant nutrients to available form [14]. These results are in conformity with Hao et al. [15] who observed that application of inorganic fertilizer alone did not significantly improve TOC content as compared to the control while the application of inorganic fertilizer along with manure or straw significantly increased TOC content. Kaur et al. [16] reported that continuous cropping with and without use of chemical fertilizers and FYM resulted in the improvement of TOC content in a long-term CT experiment. While intensive tillage practices enhance the decomposition of SOM, no-till practices generally enhance the TOC concentration in surface soil. No-till provides greater physical protection to TOC within macro-aggregate protected TOC than with CT but mostly at soil surface [17]. The higher TOC content and 
Table 8. Proportion of water soluble C (WSC), particulate organic C (POC) and light fraction organic C (LFOC) in total organic C (TOC), and particulate organic $N$ (PON) and light fraction organic $N$ (LFON) in total N (TN) in $0-5 \mathrm{~cm}$ soil layer after 2 and 4 years of soybean-wheat rotation as influenced by fertilizers, FYM and crop residue management practices under conventional tillage (CT) and conservation agriculture (CA).

\begin{tabular}{|c|c|c|c|c|c|c|c|c|c|c|c|c|}
\hline \multirow[t]{2}{*}{ Treatment No. } & \multicolumn{2}{|l|}{ Treatments } & \multicolumn{2}{|c|}{ WSC ( $\%$ of TOC) } & \multicolumn{2}{|c|}{ POC (\% of TOC) } & \multicolumn{2}{|c|}{ LFOC ( $\%$ of TOC) } & \multicolumn{2}{|c|}{$\mathrm{PON}(\%$ of $\mathrm{TN})$} & \multicolumn{2}{|c|}{ LFON ( $\%$ of TN $)$} \\
\hline & Soybean & Wheat & 2 -year & 4-year & 2-year & 4-year & 2-year & 4-year & 2 -year & 4-year & 2-year & 4-year \\
\hline \multicolumn{13}{|c|}{ Conventional tillage } \\
\hline $\mathrm{T}_{1}$ & $\mathrm{~N}_{0} \mathrm{P}_{0} \mathrm{WR}_{0}^{\dagger}$ & $\mathrm{N}_{0} \mathrm{P}_{0} \mathrm{SR}_{0}^{\ddagger}$ & 0.29 & 0.32 & 10.2 & 10.5 & 1.0 & 1.1 & 4.0 & 4.0 & 1.3 & 1.3 \\
\hline $\mathrm{T}_{2}$ & $\mathrm{~N}_{20} \mathrm{P}_{60} \mathrm{WR}_{0}$ & $\mathrm{~N}_{120} \mathrm{P}_{60} \mathrm{SR}_{0}$ & 0.39 & 0.38 & 13.1 & 14.9 & 1.8 & 1.8 & 11.6 & 11.9 & 1.7 & 1.8 \\
\hline $\mathrm{T}_{3}$ & $\mathrm{~N}_{25} \mathrm{P}_{75} \mathrm{WR}_{0}$ & $\mathrm{~N}_{150} \mathrm{P}_{75} \mathrm{SR}_{0}$ & 0.44 & 0.47 & 14.9 & 15.8 & 2.2 & 2.2 & 14.6 & 14.6 & 1.8 & 2.0 \\
\hline $\mathrm{T}_{4}$ & $\mathrm{~N}_{20} \mathrm{P}_{60} \mathrm{WR}_{0}+\mathrm{FYM}_{10} \hat{}$ & $\mathrm{N}_{120} \mathrm{P}_{60} \mathrm{SR}_{0}$ & 0.51 & 0.43 & 17.5 & 15.0 & 3.3 & 2.7 & 16.6 & 16.9 & 2.2 & 2.4 \\
\hline $\mathrm{T}_{5}$ & $\mathrm{~N}_{0} \mathrm{P}_{0} \mathrm{WR}_{6}$ & $\mathrm{~N}_{0} \mathrm{P}_{0} \mathrm{SR}_{3}$ & 0.31 & 0.31 & 12.4 & 13.7 & 1.6 & 1.4 & 4.2 & 4.3 & 1.8 & 1.9 \\
\hline $\mathrm{T}_{6}$ & $\mathrm{~N}_{20} \mathrm{P}_{60} \mathrm{WR}_{6}$ & $\mathrm{~N}_{120} \mathrm{P}_{60} \mathrm{SR}_{3}$ & 0.46 & 0.45 & 20.9 & 19.5 & 2.1 & 1.9 & 11.8 & 11.7 & 1.7 & 1.7 \\
\hline $\mathrm{T}_{7}$ & $\mathrm{~N}_{25} \mathrm{P}_{75} \mathrm{WR}_{6}$ & $\mathrm{~N}_{150} \mathrm{P}_{75} \mathrm{SR}_{3}$ & 0.43 & 0.42 & 21.5 & 19.5 & 2.7 & 2.5 & 13.6 & 13.8 & 1.9 & 2.0 \\
\hline $\mathrm{T}_{8}$ & $\mathrm{~N}_{20} \mathrm{P}_{60} \mathrm{WR}_{6}+\mathrm{FYM}_{10}$ & $\mathrm{~N}_{120} \mathrm{P}_{60} \mathrm{SR}_{3}$ & 0.49 & 0.44 & 22.6 & 22.6 & 3.0 & 2.9 & 12.1 & 12.3 & 1.7 & 1.7 \\
\hline & CT Mean & & 0.42 & 0.40 & 16.7 & 16.4 & 2.2 & 2.1 & 11.0 & 11.2 & 1.8 & 1.9 \\
\hline \multicolumn{13}{|c|}{ Conservation agriculture } \\
\hline $\mathrm{T}_{9}$ & $\mathrm{~N}_{0} \mathrm{P}_{0} \mathrm{WR}_{0}$ & $\mathrm{~N}_{0} \mathrm{P}_{0} \mathrm{SR}_{0}$ & 0.44 & 0.46 & 11.0 & 11.4 & 1.0 & 1.1 & 4.9 & 4.9 & 1.3 & 1.4 \\
\hline $\mathrm{T}_{10}$ & $\mathrm{~N}_{20} \mathrm{P}_{60} \mathrm{WR}_{0}$ & $\mathrm{~N}_{120} \mathrm{P}_{60} \mathrm{SR}_{0}$ & 0.52 & 0.52 & 14.6 & 15.0 & 1.8 & 1.9 & 13.0 & 12.9 & 1.7 & 1.8 \\
\hline $\mathrm{T}_{11}$ & $\mathrm{~N}_{25} \mathrm{P}_{75} \mathrm{WR}_{0}$ & $\mathrm{~N}_{150} \mathrm{P}_{75} \mathrm{SR}_{0}$ & 0.55 & 0.53 & 15.2 & 16.1 & 2.5 & 2.4 & 15.5 & 15.8 & 2.1 & 2.2 \\
\hline $\mathrm{T}_{12}$ & $\mathrm{~N}_{20} \mathrm{P}_{60} \mathrm{WR}_{0}+\mathrm{FYM}_{10}$ & $\mathrm{~N}_{120} \mathrm{P}_{60} \mathrm{SR}_{0}$ & 0.54 & 0.47 & 18.1 & 15.8 & 3.5 & 3.0 & 18.2 & 18.6 & 2.2 & 2.2 \\
\hline $\mathrm{T}_{13}$ & $\mathrm{~N}_{0} \mathrm{P}_{0} \mathrm{WR}_{6}$ & $\mathrm{~N}_{0} \mathrm{P}_{0} \mathrm{SR}_{3}$ & 0.48 & 0.51 & 12.8 & 14.2 & 1.4 & 1.4 & 5.2 & 5.0 & 1.7 & 1.7 \\
\hline $\mathrm{T}_{14}$ & $\mathrm{~N}_{20} \mathrm{P}_{60} \mathrm{WR}_{6}$ & $\mathrm{~N}_{120} \mathrm{P}_{60} \mathrm{SR}_{3}$ & 0.53 & 0.55 & 21.2 & 23.0 & 2.1 & 2.1 & 12.6 & 12.7 & 1.7 & 1.8 \\
\hline $\mathrm{T}_{15}$ & $\mathrm{~N}_{25} \mathrm{P}_{75} \mathrm{WR}_{6}$ & $\mathrm{~N}_{150} \mathrm{P}_{75} \mathrm{SR}_{3}$ & 0.54 & 0.49 & 21.9 & 22.3 & 2.8 & 2.5 & 15.1 & 15.0 & 1.9 & 2.0 \\
\hline \multirow[t]{3}{*}{$\mathrm{T}_{16}$} & $\mathrm{~N}_{20} \mathrm{P}_{60} \mathrm{WR}_{6}+\mathrm{FYM}_{10}$ & $\mathrm{~N}_{120} \mathrm{P}_{60} \mathrm{SR}_{3}$ & 0.55 & 0.49 & 27.2 & 24.2 & 3.6 & 3.2 & 13.8 & 14.3 & 1.6 & 1.6 \\
\hline & CA Mean & & 0.52 & 0.50 & 17.8 & 17.7 & 2.4 & 2.2 & 12.3 & 12.4 & 1.8 & 1.8 \\
\hline & Overall mean & & 0.47 & 0.45 & 17.2 & 17.1 & 2.3 & 2.1 & 11.7 & 11.8 & 1.8 & 1.8 \\
\hline
\end{tabular}

${ }^{\dagger} \mathrm{N}=$ fertilizer $\mathrm{N}\left(\mathrm{kg} \mathrm{N} \mathrm{ha}^{-1}\right) ; \mathrm{P}=$ fertilizer $\mathrm{P}\left(\mathrm{kg} \mathrm{P}_{2} \mathrm{O}_{5} \mathrm{ha}^{-1}\right) ; \mathrm{WR}=$ Wheat crop residue $\left(\mathrm{t} \cdot \mathrm{ha}{ }^{-1}\right) ;{ }^{\dagger} \mathrm{SR}=\mathrm{Soybean}$ crop residue $\left(\mathrm{t} \cdot \mathrm{ha}{ }^{-1}\right) ;{ }^{\wedge} \mathrm{FYM}=$ farmyard manure $\left(\mathrm{t} \cdot \mathrm{ha}^{-1}\right)$.

stock found in CA were due to better preservation of the SOM originally present in the soil and/or less mineralization of surface retained organic residues [18].

After 4 years of study, maximum WSC contents of 25.8 and $29.7 \mathrm{mg} \cdot \mathrm{kg}^{-1}$ were found in $0-5 \mathrm{~cm}$ and 19.2 and $26.4 \mathrm{mg} \cdot \mathrm{kg}^{-1}$ in $5-15 \mathrm{~cm}$ soil layer in $\mathrm{T}_{8}$ and $\mathrm{T}_{16}$ treatments in CT and CA, respectively (Tables 5 and 6). Same treatment resulted in the maximum contents of POC, PON, LFOC, LFON, PMN, MBC and MBN in CT and $\mathrm{CA}$ and the effect of tillage was statistically significant (Tables 5-7). Thus, integrated use of inorganic fertilizers, FYM and CR significantly improved these labile $\mathrm{C}$ and $\mathrm{N}$ pools of soil.

The proportions of WSC, POC, LFOC and MBC in TOC and of PON, PMN and MBN in TN were highest in CA system (Tables 8-10). Significantly higher contents and proportions of these labile $\mathrm{C}$ and $\mathrm{N}$ pools obtained with CA than CT were more pronounced in $0-5 \mathrm{~cm}$ soil layer. These results indicated that POC, LFOC MBC, PON, PMN, MBN and LFOC can be used as sensitive indicators of management effects. Increased $\mathrm{MBC}$ in TOC and MBN in TN also with the addition of fertilizer and organic sources could be attributed to the better crop growth resulting in greater root derived organic matter. The significant increase in $\mathrm{MBC}$ in treatments containing FYM + CR could be ascribed to the availability of more carbon as was evident from several other fractions of TOC such as WSC, POM, LFOC, and MBC. The rapid buildup of microbial biomass in subtropical conditions implies that MBN could serve as a potential source of mineralizable $\mathrm{N}$ for plant nutrition in such soils. $\mathrm{MBC}$ is an active component of SOM and constitutes an important soil 
Table 9. Proportion of water soluble C (WSC), particulate organic C (POC) and light fraction organic C (LFOC) in total organic C (TOC), and particulate organic N (PON) and light fraction organic N (LFON) in total N (TN) in 5 - $15 \mathrm{~cm}$ soil layer after 2 and 4 years of soybean-wheat rotation as influenced by fertilizers, FYM and crop residue management practices under conventional tillage (CT) and conservation agriculture (CA).

\begin{tabular}{|c|c|c|c|c|c|c|c|c|c|c|c|c|}
\hline \multirow[t]{2}{*}{ Treatment No. } & \multicolumn{2}{|c|}{ Treatments } & \multicolumn{2}{|c|}{ WSC ( $\%$ of TOC) } & \multicolumn{2}{|c|}{ POC (\% of TOC) } & \multicolumn{2}{|c|}{ LFOC ( $\%$ of TOC) } & \multicolumn{2}{|c|}{ PON (\% of TN) } & \multicolumn{2}{|c|}{ LFON ( $\%$ of TN $)$} \\
\hline & Soybean & Wheat & 2-year & 4 -year & 2-year & 4-year & 2-year & 4-year & 2 -year & 4-year & 2-year & 4-year \\
\hline \multicolumn{13}{|c|}{ Conventional tillage } \\
\hline $\mathrm{T}_{1}$ & $\mathrm{~N}_{0} \mathrm{P}_{0} \mathrm{WR}_{0}^{\dagger}$ & $\mathrm{N}_{0} \mathrm{P}_{0} \mathrm{SR}_{0}^{\ddagger}$ & 0.32 & 0.37 & 9.7 & 11.4 & 1.2 & 1.2 & 3.7 & 3.8 & 1.2 & 1.3 \\
\hline $\mathrm{T}_{2}$ & $\mathrm{~N}_{20} \mathrm{P}_{60} \mathrm{WR}_{0}$ & $\mathrm{~N}_{120} \mathrm{P}_{60} \mathrm{SR}_{0}$ & 0.35 & 0.40 & 13.7 & 15.8 & 1.9 & 2.0 & 9.9 & 10.4 & 1.6 & 1.7 \\
\hline $\mathrm{T}_{3}$ & $\mathrm{~N}_{25} \mathrm{P}_{75} \mathrm{WR}_{0}$ & $\mathrm{~N}_{150} \mathrm{P}_{75} \mathrm{SR}_{0}$ & 0.39 & 0.39 & 16.4 & 15.2 & 2.5 & 2.3 & 12.7 & 13.1 & 1.9 & 2.1 \\
\hline $\mathrm{T}_{4}$ & $\mathrm{~N}_{20} \mathrm{P}_{60} \mathrm{WR}_{0}+\mathrm{FYM}_{10} \hat{~}$ & $\mathrm{~N}_{120} \mathrm{P}_{60} \mathrm{SR}_{0}$ & 0.41 & 0.45 & 17.3 & 18.6 & 3.7 & 3.5 & 14.6 & 14.4 & 1.9 & 1.9 \\
\hline $\mathrm{T}_{5}$ & $\mathrm{~N}_{0} \mathrm{P}_{0} \mathrm{WR}_{6}$ & $\mathrm{~N}_{0} \mathrm{P}_{0} \mathrm{SR}_{3}$ & 0.35 & 0.36 & 11.4 & 11.1 & 1.7 & 1.5 & 3.5 & 3.6 & 1.5 & 1.7 \\
\hline $\mathrm{T}_{6}$ & $\mathrm{~N}_{20} \mathrm{P}_{60} \mathrm{WR}_{6}$ & $\mathrm{~N}_{120} \mathrm{P}_{60} \mathrm{SR}_{3}$ & 0.39 & 0.39 & 18.0 & 19.2 & 2.0 & 1.9 & 10.6 & 11.7 & 1.2 & 1.4 \\
\hline $\mathrm{T}_{7}$ & $\mathrm{~N}_{25} \mathrm{P}_{75} \mathrm{WR}_{6}$ & $\mathrm{~N}_{150} \mathrm{P}_{75} \mathrm{SR}_{3}$ & 0.42 & 0.42 & 18.7 & 17.4 & 2.6 & 2.2 & 13.5 & 13.6 & 1.5 & 1.7 \\
\hline $\mathrm{T}_{8}$ & $\mathrm{~N}_{20} \mathrm{P}_{60} \mathrm{WR}_{6}+\mathrm{FYM}_{10}$ & $\mathrm{~N}_{120} \mathrm{P}_{60} \mathrm{SR}_{3}$ & 0.45 & 0.46 & 19.6 & 20.1 & 3.5 & 3.0 & 12.1 & 12.1 & 1.3 & 1.3 \\
\hline & CT Mean & & 0.39 & 0.40 & 15.6 & 16.1 & 2.4 & 2.2 & 10.1 & 10.3 & 1.5 & 1.6 \\
\hline \multicolumn{13}{|c|}{ Conservation agriculture } \\
\hline $\mathrm{T}_{9}$ & $\begin{array}{lll}\mathrm{N}_{0} & \mathrm{P}_{0} & \mathrm{WR}_{0}\end{array}$ & $\begin{array}{lll}\mathrm{N}_{0} & \mathrm{P}_{0} & \mathrm{SR}_{0}\end{array}$ & 0.34 & 0.35 & 10.3 & 11.5 & 1.2 & 1.2 & 4.9 & 5.0 & 1.3 & 1.4 \\
\hline $\mathrm{T}_{10}$ & $\mathrm{~N}_{20} \mathrm{P}_{60} \mathrm{WR}_{0}$ & $\mathrm{~N}_{120} \mathrm{P}_{60} \mathrm{SR}_{0}$ & 0.41 & 0.46 & 14.1 & 15.6 & 2.1 & 2.1 & 12.8 & 12.8 & 1.7 & 1.8 \\
\hline $\mathrm{T}_{11}$ & $\mathrm{~N}_{25} \mathrm{P}_{75} \mathrm{WR}_{0}$ & $\mathrm{~N}_{150} \mathrm{P}_{75} \mathrm{SR}_{0}$ & 0.40 & 0.55 & 16.9 & 17.7 & 3.0 & 3.0 & 14.8 & 14.6 & 2.2 & 2.4 \\
\hline $\mathrm{T}_{12}$ & $\mathrm{~N}_{20} \mathrm{P}_{60} \mathrm{WR}_{0}+\mathrm{FYM}_{10}$ & $\mathrm{~N}_{120} \mathrm{P}_{60} \mathrm{SR}_{0}$ & 0.57 & 0.59 & 17.9 & 18.8 & 4.5 & 4.3 & 19.1 & 19.1 & 2.3 & 2.3 \\
\hline $\mathrm{T}_{13}$ & $\mathrm{~N}_{0} \mathrm{P}_{0} \mathrm{WR}_{6}$ & $\mathrm{~N}_{0} \mathrm{P}_{0} \mathrm{SR}_{3}$ & 0.38 & 0.38 & 12.1 & 12.6 & 1.7 & 1.7 & 4.8 & 4.9 & 1.7 & 1.8 \\
\hline $\mathrm{T}_{14}$ & $\mathrm{~N}_{20} \mathrm{P}_{60} \mathrm{WR}_{6}$ & $\mathrm{~N}_{120} \mathrm{P}_{60} \mathrm{SR}_{3}$ & 0.42 & 0.43 & 18.4 & 17.9 & 2.4 & 2.3 & 12.3 & 12.2 & 1.5 & 1.6 \\
\hline $\mathrm{T}_{15}$ & $\mathrm{~N}_{25} \mathrm{P}_{75} \mathrm{WR}_{6}$ & $\mathrm{~N}_{150} \mathrm{P}_{75} \mathrm{SR}_{3}$ & 0.50 & 0.52 & 19.3 & 20.3 & 3.0 & 2.8 & 15.6 & 15.2 & 1.8 & 1.9 \\
\hline \multirow[t]{2}{*}{$\mathrm{T}_{16}$} & $\mathrm{~N}_{20} \mathrm{P}_{60} \mathrm{WR}_{6}+\mathrm{FYM}_{10}$ & $\mathrm{~N}_{120} \mathrm{P}_{60} \mathrm{SR}_{3}$ & 0.69 & 0.60 & 20.7 & 18.6 & 4.1 & 3.5 & 17.0 & 18.1 & 1.8 & 1.9 \\
\hline & CA Mean & & 0.46 & 0.48 & 16.2 & 16.6 & 2.7 & 2.6 & 12.7 & 12.8 & 1.8 & 1.9 \\
\hline & Overall mean & & 0.43 & 0.44 & 15.9 & 16.4 & 2.6 & 2.4 & 11.4 & 11.5 & 1.7 & 1.8 \\
\hline
\end{tabular}

$\overline{{ }^{\dagger} \mathrm{N}}=$ fertilizer $\mathrm{N}\left(\mathrm{kg} \mathrm{N} \mathrm{ha}^{-1}\right) ; \mathrm{P}=$ fertilizer $\mathrm{P}\left(\mathrm{kg} \mathrm{P}_{2} \mathrm{O}_{5} \mathrm{ha}^{-1}\right) ; \mathrm{WR}=$ Wheat crop residue $\left(\mathrm{t} \cdot \mathrm{ha}^{-1}\right) ;{ }^{\dagger} \mathrm{SR}=\mathrm{Soybean}$ crop residue $\left(\mathrm{t} \cdot \mathrm{ha}{ }^{-1}\right) ;{ }^{\wedge} \mathrm{FYM}=$ farmyard manure $\left(\mathrm{t} \cdot \mathrm{ha}^{-1}\right)$.

health parameter as carbon contained within microbial biomass is a stored energy for microbial process. Thus $\mathrm{MBC}$ and $\mathrm{MBN}$, the measure of potential microbial activity, are strongly related to soil aggregate stability. POM, dominated by undecomposed plant residues that retain recognizable cell structures including fungal hyphae, seeds, spores, and fungal skeletons, is an active fraction of SOM, which supplies nutrients to the growing plants [19]. POM-C and POM-N provide estimates of the intermediate pool of SOM between the active and passive pools [8] and provide substrate for microorganisms and influence soil aggregation [20,21]. LFOM, composed primarily of plant derived remains, and microbial and micro-faunal debris and other incompletely decomposed organic residues, is more sensitive to management prac- tices than POM [22].

PMN, a measure of the soil capacity to supply mineral $\mathrm{N}$, constitutes an important measure of the soil health due to its strong relationship with the capability of soil to supply $\mathrm{N}$ for crop growth. In an earlier study from Ludhiana, Tirol-Padre et al. [23] observed that PMN was highest with GM+NPK under anaerobic incubation. In another study from Ludhiana, Walia and Kler [24] revealed higher mineralizable $\mathrm{N}$ under organic farming treatments as compared to chemical fertilizers alone showing better availability of $\mathrm{N}$ under organic farming. Kang et al. [25] found that application of organic residues increased PMN, which was positively related to increase in TOC content of soil. Yan et al. [26] reported that response of the LFOC and LFON contents to fertilization 
Table 10. Proportion of microbial biomass C (MBC) in total organic C (TOC), and potentially mineralizable N (PMN) and microbial biomass $N(M B N)$ in total $N(T N)$ in soil after 2 and 4 years of soybean-wheat rotation as influenced by fertilizers, FYM and crop residue management practices under conventional tillage (CT) and conservation agriculture (CA).

\begin{tabular}{|c|c|c|c|c|c|c|c|c|c|c|}
\hline \multirow{3}{*}{$\begin{array}{c}\text { Treatment } \\
\text { No. }\end{array}$} & \multicolumn{2}{|c|}{ Treatments } & \multicolumn{4}{|c|}{ PMN (\% of TN) } & \multirow{2}{*}{\multicolumn{2}{|c|}{$\begin{array}{c}\begin{array}{c}\text { MBC } \\
(\% \text { of TOC })\end{array} \\
0-5 \mathrm{~cm} \text { layer }\end{array}$}} & \multirow{2}{*}{\multicolumn{2}{|c|}{$\begin{array}{c}\begin{array}{c}\text { MBN } \\
(\% \text { of TN })\end{array} \\
0-5 \text { cm layeı }\end{array}$}} \\
\hline & \multirow[t]{2}{*}{ Soybean } & \multirow[t]{2}{*}{ Wheat } & \multicolumn{2}{|c|}{$0-5 \mathrm{~cm}$ layer } & \multicolumn{2}{|c|}{$5-15 \mathrm{~cm}$ layer } & & & & \\
\hline & & & $2-y r$ & $4-y r$ & $2-y r$ & $4-y r$ & $2-y r$ & $4-y r$ & $2-y r$ & $4-y r$ \\
\hline & Conventional tillage & & & & & & & & & \\
\hline $\mathrm{T}_{1}$ & $\mathrm{~N}_{0} \mathrm{P}_{0} \mathrm{WR}_{0}^{\dagger}$ & $\mathrm{N}_{0} \mathrm{P}_{0} \mathrm{SR}_{0}{ }^{\star}$ & 0.61 & 0.74 & 0.33 & 0.38 & 2.4 & 2.4 & 1.4 & 1.4 \\
\hline $\mathrm{T}_{2}$ & $\mathrm{~N}_{20} \mathrm{P}_{60} \mathrm{WR}_{0}$ & $\mathrm{~N}_{120} \mathrm{P}_{60} \mathrm{SR}_{0}$ & 0.57 & 0.66 & 0.61 & 0.65 & 3.0 & 3.1 & 1.9 & 1.9 \\
\hline $\mathrm{T}_{3}$ & $\mathrm{~N}_{25} \mathrm{P}_{75} \mathrm{WR}_{0}$ & $\mathrm{~N}_{150} \mathrm{P}_{75} \mathrm{SR}_{0}$ & 0.71 & 0.79 & 0.79 & 0.81 & 3.1 & 3.2 & 2.3 & 2.5 \\
\hline $\mathrm{T}_{4}$ & $\mathrm{~N}_{20} \mathrm{P}_{60} \mathrm{WR}_{0}+\mathrm{FYM}_{10} \hat{~}$ & $\mathrm{~N}_{120} \mathrm{P}_{60} \mathrm{SR}_{0}$ & 0.88 & 0.91 & 0.85 & 0.85 & 3.5 & 2.8 & 2.7 & 2.9 \\
\hline $\mathrm{T}_{5}$ & $\mathrm{~N}_{0} \mathrm{P}_{0} \mathrm{WR}_{6}$ & $\mathrm{~N}_{0} \mathrm{P}_{0} \mathrm{SR}_{3}$ & 0.70 & 0.72 & 0.38 & 0.39 & 2.9 & 2.8 & 1.5 & 1.5 \\
\hline $\mathrm{T}_{6}$ & $\mathrm{~N}_{20} \mathrm{P}_{60} \mathrm{WR}_{6}$ & $\mathrm{~N}_{120} \mathrm{P}_{60} \mathrm{SR}_{3}$ & 1.18 & 1.20 & 1.02 & 1.07 & 3.3 & 3.0 & 2.2 & 2.2 \\
\hline $\mathrm{T}_{7}$ & $\mathrm{~N}_{25} \mathrm{P}_{75} \mathrm{WR}_{6}$ & $\mathrm{~N}_{150} \mathrm{P}_{75} \mathrm{SR}_{3}$ & 1.28 & 1.33 & 1.12 & 1.16 & 3.4 & 3.5 & 2.7 & 2.7 \\
\hline \multirow[t]{3}{*}{$\mathrm{T}_{8}$} & $\mathrm{~N}_{20} \mathrm{P}_{60} \mathrm{WR}_{6}+\mathrm{FYM}_{10}$ & $\mathrm{~N}_{120} \mathrm{P}_{60} \mathrm{SR}_{3}$ & 1.15 & 1.23 & 1.13 & 1.16 & 3.5 & 3.3 & 2.9 & 3.0 \\
\hline & CT Mean & & 0.88 & 0.95 & 0.78 & 0.81 & 3.2 & 3.0 & 2.2 & 2.3 \\
\hline & \multicolumn{10}{|c|}{ Conservation agriculture } \\
\hline $\mathrm{T}_{9}$ & $\mathrm{~N}_{0} \mathrm{P}_{0} \mathrm{WR}_{0}$ & $\mathrm{~N}_{0} \mathrm{P}_{0} \mathrm{SR}_{0}$ & 0.76 & 0.79 & 0.35 & 0.42 & 2.5 & 2.6 & 1.4 & 1.4 \\
\hline $\mathrm{T}_{10}$ & $\mathrm{~N}_{20} \mathrm{P}_{60} \mathrm{WR}_{0}$ & $\mathrm{~N}_{120} \mathrm{P}_{60} \mathrm{SR}_{0}$ & 0.71 & 0.73 & 0.47 & 0.50 & 3.0 & 3.0 & 1.9 & 1.9 \\
\hline $\mathrm{T}_{11}$ & $\mathrm{~N}_{25} \mathrm{P}_{75} \mathrm{WR}_{0}$ & $\mathrm{~N}_{150} \mathrm{P}_{75} \mathrm{SR}_{0}$ & 0.89 & 0.94 & 0.73 & 0.74 & 3.2 & 3.1 & 2.7 & 2.8 \\
\hline $\mathrm{T}_{12}$ & $\mathrm{~N}_{20} \mathrm{P}_{60} \mathrm{WR}_{0}+\mathrm{FYM}_{10}$ & $\mathrm{~N}_{120} \mathrm{P}_{60} \mathrm{SR}_{0}$ & 1.06 & 1.13 & 0.98 & 1.01 & 3.4 & 2.9 & 2.7 & 2.7 \\
\hline $\mathrm{T}_{13}$ & $\mathrm{~N}_{0} \mathrm{P}_{0} \mathrm{WR}_{6}$ & $\mathrm{~N}_{0} \mathrm{P}_{0} \mathrm{SR}_{3}$ & 0.80 & 0.89 & 0.60 & 0.66 & 2.8 & 2.9 & 1.4 & 1.5 \\
\hline $\mathrm{T}_{14}$ & $\mathrm{~N}_{20} \mathrm{P}_{60} \mathrm{WR}_{6}$ & $\mathrm{~N}_{120} \mathrm{P}_{60} \mathrm{SR}_{3}$ & 1.38 & 1.46 & 0.97 & 1.11 & 3.2 & 3.2 & 2.4 & 2.5 \\
\hline $\mathrm{T}_{15}$ & $\mathrm{~N}_{25} \mathrm{P}_{75} \mathrm{WR}_{6}$ & $\mathrm{~N}_{150} \mathrm{P}_{75} \mathrm{SR}_{3}$ & 1.50 & 1.59 & 1.33 & 1.33 & 3.3 & 3.4 & 3.0 & 3.3 \\
\hline \multirow[t]{3}{*}{$\mathrm{T}_{16}$} & $\mathrm{~N}_{20} \mathrm{P}_{60} \mathrm{WR}_{6}+\mathrm{FYM}_{10}$ & $\mathrm{~N}_{120} \mathrm{P}_{60} \mathrm{SR}_{3}$ & 1.37 & 1.42 & 1.58 & 1.65 & 4.3 & 3.6 & 3.1 & 2.9 \\
\hline & CA Mean & & 1.06 & 1.12 & 0.87 & 0.93 & 3.2 & 3.1 & 2.3 & 2.4 \\
\hline & Overall mean & & 0.97 & 1.03 & 0.83 & 0.87 & 3.2 & 3.1 & 2.3 & 2.3 \\
\hline
\end{tabular}

${ }^{\dagger} \mathrm{N}=$ fertilizer $\mathrm{N}\left(\mathrm{kg} \mathrm{N} \mathrm{ha}^{-1}\right) ; \mathrm{P}=$ fertilizer $\mathrm{P}\left(\mathrm{kg} \mathrm{P}_{2} \mathrm{O}_{5} \mathrm{ha}^{-1}\right) ; \mathrm{WR}=$ Wheat crop residue $\left(\mathrm{t} \cdot \mathrm{ha}{ }^{-1}\right) ;{ }^{\dagger} \mathrm{SR}=$ Soybean crop residue $\left(\mathrm{t} \cdot \mathrm{ha}{ }^{-1}\right) ;{ }^{\wedge} \mathrm{FYM}=$ farmyard manure $\left(\mathrm{t} \cdot \mathrm{ha}^{-1}\right)$.

treatments was similar to those observed for the POC and PON contents. Manna et al. [27] also observed that LFOC and LFON did not show any significant changes with $\mathrm{N}, \mathrm{N}+\mathrm{P}$ and $\mathrm{N}+\mathrm{P}+\mathrm{K}$ treatments except $\mathrm{N}+\mathrm{P}+$ $\mathrm{K}+\mathrm{FYM}$ treatment because newly humified organic matter application through FYM sustained higher amounts of LFOC and LFON content. Dalal et al. [28] studied the effects of 20 years of tillage practice, CR management and fertilizer $\mathrm{N}$ application on microbial biomass and found that $\mathrm{MBN}$ was significantly affected by tillage, residue and fertilizer $\mathrm{N}$ individually as well as through their interaction. The soil layers under no-till contained higher amount of MBN than that under CT treatments.

Thus, application of inorganic fertilizers like $\mathrm{N}$ and $\mathrm{P}$ in optimum amount along with organic manures and crop residue incorporation provides a better option in signifi- cantly improving the aggregate stability, MWD, storage of nutrients in labile pools, and $\mathrm{C}$ sequestration in semiarid subtropical soils that are inherently low in organic matter and nutrients.

\section{Conclusion}

Soil conservation management improved the quality of the soil by enhancing the labile and total organic carbon fractions and biological status, especially in $0-5 \mathrm{~cm}$ upper layer. Results of this 4-year field study with soybean-wheat cropping rotation indicate that the content of TOC, POM-C, POM-N, LFOM-C, LFOM-N and PMN decreased with soil depth, and thin surface layer (0 - 5 $\mathrm{cm}$ ) contained much higher concentration of these labile pools than 5 - $15 \mathrm{~cm}$ subsurface layer. The surface soil layer had substantially higher levels of all soil health 
parameters than subsurface layer, presumably due to higher retention of crop stubbles, fallen leaves and root biomass. The enhanced proportions of WSC, POM-C, LFOM-C, MBC in TOC and that of POM-N, LFOM-N, MBN in TN with the supply of optimum and balanced N, $\mathrm{P}$ and organic manures and incorporation of crop residues indicate that the improvement in labile forms of both $\mathrm{C}$ and $\mathrm{N}$ was relatively rapid than control suggesting that active $\mathrm{C}$ and $\mathrm{N}$ pools reflect changes due to integrated nutrient management (INM). INM significantly increased water stable aggregates and had profound effects in increasing the mean weight diameter as well as the formation of macro-aggregates, which were highest in both surface $(85 \%)$ and subsurface $(81 \%)$ soil layers with application of $20 \mathrm{~kg} \mathrm{~N}+60 \mathrm{~kg} \mathrm{P}_{2} \mathrm{O}_{5}+10 \mathrm{t} \mathrm{FYM} \mathrm{+6}$ t WR ha ${ }^{-1}$ applied to soybean and $120 \mathrm{~kg} \mathrm{~N}+60 \mathrm{~kg} \mathrm{P}_{2} \mathrm{O}_{5}$ $+3 \mathrm{t} \mathrm{SR} \mathrm{ha}^{-1}$ applied to wheat crops in CA $\left(\mathrm{T}_{16}\right)$ and respectively, $83 \%$ and $77 \%$ in $\mathrm{CT}\left(\mathrm{T}_{8}\right)$ treatments after 2 cycles of the experiment. Similarly, the content and stock of TOC and contents of POM-C, LFOC-C, PMN, MBC and MBN were highest with the INM. Thus, application of fertilizer in optimum amounts and inclusion of manure in the fertilizer schedule could maintain the soil health under intensive agriculture. In conclusion, INM plays a significant role in building-up/restoring soil health and productivity with co-benefits of improved $\mathrm{C}$ sequestration in semiarid subtropical soils inherently low in organic matter and nutrients.

\section{Acknowledgements}

The financial support provided by FAO/IAEA Coordinated Research Project D15009 on "Integrated soil, water and nutrient management for conservation agriculture", through Research Contract No. IND/12980 is gratefully acknowledged by the authors.

\section{REFERENCES}

[1] R. T. Conant, M. Easter, K. Paustian, A. Swan and S. Williams, "Impacts of Periodic Tillage on Soil C Stocks: A Synthesis," Soil Tillage and Research, Vol. 95, No. 1-2, 2007, pp. 1-10. http://dx.doi.org/10.1016/j.still.2006.12.006

[2] S. Kumar, M. S. Aulakh and A. K. Garg, "Soil Aggregates, Organic Matter, and Labile C and N Fractions after 37 Years of N, P and K Applications to an Irrigated Subtropical Soil under Maize-Wheat Rotation," Journal of Agricultural Science and Technology, Vol. 1, No. 2A, 2011, pp. 170-181.

[3] K. K. M. Nambiar, "Major Cropping Systems in India," In: Barnett, et al., Eds., Agricultural Sustainability: Economics, Environmental and Statistical Considerations, John Wiley \& Sons, Chichester, 1995, pp. 135-142.

[4] M. S. Aulakh, N. S. Pasricha and G. S. Bahl, "Phosphorus Fertilizer Response in an Irrigated Soybean-Wheat Pro- duction System on a Subtropical, Semiarid Soil," Field Crops Research, Vol. 80, No. 2, 2003, pp. 99-109. http://dx.doi.org/10.1016/S0378-4290(02)00172-7

[5] M. S. Aulakh, J. S. Manchanda, A. K. Garg, S. Kumar, G. Dercon and M. L. Nguyen, "Crop production and Nutrient Use Efficiency of Conservation Agriculture for SoybeanWheat Rotation in the Indo-Gangetic Plains of Northwestern India," Soil Tillage and Research, Vol. 120, 2012, pp. 50-60. http://dx.doi.org/10.1016/j.still.2011.11.001

[6] R. E. Yoder, "A Direct Method of Aggregate Analysis of Soils and a Study of the Physical Nature of Soil Erosion Losses," Journal of American Society of Agronomy, Vol. 28, No. 5, 1936, pp. 337-351. http://dx.doi.org/10.2134/agronj1936.0002196200280005 $\underline{0001 x}$

[7] W. B. McGill, K. R. Cannon, J. A. Robertson and F. D. Cook, "Dynamics of Soil Microbial Biomass and Water Soluble Organic C in Breton L after 50 Years of Cropping to Two Rotations," Canadian Journal of Soil Science, Vol. 66, No. 1, 1986, pp. 1-19. http://dx.doi.org/10.4141/cjss86-001

[8] C. A. Cambardella and E. T. Elliott, "Particulate Soil Organic Matter Changes across a Grassland Cultivation Sequence," Soil Science Society of America Journal, Vol. 56, No. 3, 1992, pp. 777-783. http://dx.doi.org/10.2136/sssaj1992.03615995005600030 $\underline{017 \mathrm{x}}$

[9] J. E. Compton and R. D. Boone, "Soil Nitrogen Transformation and the Role of Light Fraction Organic Matter in Forest Soils," Soil Biology and Biochemistry, Vol. 34, No. 7, 2002, pp. 933-943. http://dx.doi.org/10.1016/S0038-0717(02)00025-1

[10] D. R. Keeney, "Nitrogen-Availability Indices" In: A. L. Page, et al., Eds., Methods in Soil Analysis-Part 2, Chemical and Microbial Properties, 2nd Edition, Soil Science Society of America, Madison, 1982, pp. 711-733.

[11] W. R. Horwath and E. A. Paul, "Microbial Biomass," In: R. W. Weaver, et al., Eds., Methods of Soil AnalysisPart 2, Microbiological and Biochemical Properties, 2nd Edition, Soil Science Society of America, Madison, 1994, pp. 753-771.

[12] P. C. Brookes, A. Landman, G. Pruden and D. S. Jenkinson, "Chloroform Fumigation and the Release of Soil Nitrogen: A Rapid Direct Extraction Method to Measure Microbial Biomass Nitrogen in Soil," Soil Biology and Biochemistry, Vol. 17, No. 6, 1985, pp. 837-842. http://dx.doi.org/10.1016/0038-0717(85)90144-0

[13] W. G. Cochran and G. M. Cox, "Experimental Designs," Wiley, New York, 1950.

[14] M. S. Aulakh, T. S. Khera, J. W. Doran, Kuldip-Singh and Bijay-Singh, "Yields and Nitrogen Dynamics in a Rice-Wheat System Using Green Manure and Inorganic Fertilizer," Soil Science Society of America Journal, Vol. 64, 2000, pp. 1867-1876.

[15] X. H. Hao, S. L. Liu, J. S. Wu, R. G. Hu, C. L. Tong and Y. Y. Su, "Effect of Long-Term Application of Inorganic Fertilizer and Organic Amendments on Soil Organic Matter and Microbial Biomass in Three Subtropical 
Paddy Soils," Nutrient Cycling in Agroecosystems, Vol. 81, No. 1, 2008, pp. 17-24. http://dx.doi.org/10.1007/s10705-007-9145-Z

[16] T. Kaur, B. S. Brar and N. S. Dhillon, "Soil Organic Matter Dynamics as Affected by Long-Term Use of Organic and Inorganic Fertilizers under Maize-Wheat Cropping System," Nutrient Cycling in Agroecosystems, Vol. 81, No. 1, 2008, pp. 59-69. http://dx.doi.org/10.1007/s10705-007-9152-0

[17] A. J. Franzluebbers and M. A. Arshad, "Soil Microbial Biomass and Mineralizable Carbon of Water-Stable Aggregates," Soil Science Society of America Journal, Vol. 61, No. 4, 1997, pp. 1090-1097. http://dx.doi.org/10.2136/sssaj1997.03615995006100040 $\underline{015 \mathrm{x}}$

[18] J. Balasdent, C. Chenu and M. Balabane, "Relationship of Soil Organic Matter Dynamics to Physical Protection and Tillage," Soil Tillage and Research, Vol. 53, No. 3-4, 2000, pp. 215-230.

http://dx.doi.org/10.1016/S0167-1987(99)00107-5

[19] E. G. Gregorich and H. H. Janzen, "Storage of Soil Carbon in the Light Fraction and Macro Organic Matter," In: M. R. Carter and B. A. Stewart, Eds., Advances in Soil Science, Structure and Organic Matter Storage in Agricultural Soils, CRC Press, Lewis Publisher, Boca Raton, 1996, pp. 167-190.

[20] A. J. Franzluebbers, G. W. Langdale and H. H. Schomberg, "Soil Carbon, Nitrogen and Aggregation in Response to Type and Frequency of Tillage," Soil Science Society of America Journal, Vol. 63, No. 2, 1999, pp. 349-355. http://dx.doi.org/10.2136/sssaj1999.03615995006300020 $\underline{012 x}$

[21] J. Six, E. T. Elliott and K. Paustian, "Aggregate and Soil Organic Matter Dynamics under Conventional and NoTillage Systems," Soil Science Society of America Journal, Vol. 63, No. 5, 1999, pp. 1350-1358. http://dx.doi.org/10.2136/sssaj1999.6351350x
[22] M. R. Carter, D. A. Angers, E. G. Gregorich and M. A. Bolinder, "Characterizing Organic Matter Retention for Surface Soils in Eastern Canada Using Density and Particle Size Fractions," Canadian Journal of Soil Science, Vol. 83, No. 1, 2003, pp. 11-23. http://dx.doi.org/10.4141/S01-087

[23] A. Tirol-Padre, K. Tsuchiya, K. Inubushi and J. K. Ladha, "Organic Amendments Affect Soil Parameters in Two Long-Term Rice-Wheat Experiments," Soil Science Society of America Journal, Vol. 71, No. 2, 2007, pp. 442-452. http://dx.doi.org/10.2136/sssaj2006.0141

[24] S. S. Walia and D. S. Kler, "Effect of Farmyard Manure on Soil Properties in Maize-Wheat System," Journal of Research, Punjab Agricultural University, Vol. 43, No. 4, 2006, pp. 292-295.

[25] G. S. Kang, V. Beri, B. S. Sidhu and O. P. Rupela, "A New Index to Assess Soil Quality and Sustainability of Wheat-Based Cropping Systems," Biology and Fertility of Soils, Vol. 41, No. 6, 2005, pp. 389-398. http://dx.doi.org/10.1007/s00374-005-0857-4

[26] D. Yan, D. Wang and L. Yang, "Long-Term Effect of Chemical Fertilizer, Straw and Manure on Labile Organic Matter Fractions in a Paddy Soil," Biology and Fertility of Soils, Vol. 44, No. 1, 2007, pp. 93-101. http://dx.doi.org/10.1007/s00374-007-0183-0

[27] M. C. Manna, A. Swarup, R. H. Wanjari, Y. V. Singh, P. K. Ghosh, K. N. Singh, A. K. Tripathi and M. N. Saha, "Soil Organic Matter in West Bengal Inceptisol after 30 Years of Multiple Cropping and Fertilization," Soil Science Society of America Journal, Vol. 70, No. 1, 2006, pp. 121-129. http://dx.doi.org/10.2136/sssaj2005.0180

[28] R. C. Dalal, P. A. Henderson and J. M. Glasby, "Organic Matter and Microbial Biomass in a Vertisol after $20 \mathrm{yr}$ of Zero-Tillage," Soil Biology and Biochemistry, Vol. 23, No. 5, 1991, pp. 435-441. http://dx.doi.org/10.1016/0038-0717(91)90006-6 